\title{
On the subsonic and low transonic aerodynamic performance of the Land Speed Record car, Bloodhound LSR
}

\author{
B. Evans *, J. Townsend, O. Hassan, K. Morgan \\ Swansea University, $U K$ \\ R. Ayers, M. Chapman and A. Green \\ Bloodhound LSR \\ M. Ingham and B. Javid \\ Nuffield Trust
}

The land speed record vehicle, Bloodhound, undertook testing at subsonic and low transonic speeds (up to Mach 0.8) at Hakskeen Pan, South Africa, during October and November of 2019. A decade of CFD-led aerodynamic design had been undertaken to produce a vehicle with the aim of minimised Mach number aerodynamic dependencies and minimised overall drag. This paper sets out and explains the measured pressure distributions across a range of test runs of the vehicle in differing atmospheric and vehicle configuration conditions. It compares the measured aerodynamic performance with the various CFD models used throughout the design process showing that, whilst localised discrepancies between CFD model and real behaviour exist, overall the RANS-based CFD tools used to design the car do result in sufficiently accurate data to predict the overall vehicle performance.

The work outlined in this paper, and the conclusions and recommendations drawn, form the basis for a future record attempt and the understanding of what would be required in principle to extend the World Land Speed Record to $1,000 \mathrm{mph}$. It also provides guidance on how to effectively make use of RANS-based CFD modelling predictions for other complex, ground-interacting high speed applications.

\section{Nomenclature}

$$
\begin{array}{ll}
C_{p} & =\text { static pressure coefficient } \\
C x & =\text { force coefficient in the } x \text { direction } \\
C y & =\text { force coefficient in the } y \text { direction } \\
C_{m} & =\text { moment coefficient } \\
c & =\text { chord }
\end{array}
$$

\footnotetext{
*b.j.evans@ swansea.ac.uk, Zienkiewicz Centre for Computational Engineering, Swansea University, Bay Campus, Swansea, SA1 8EN, UK
} 


$$
\begin{array}{ll}
D & =\text { drag } \\
G & =\text { acceleration due to gravity }\left(\mathrm{m} / \mathrm{s}^{2}\right) \\
\mathrm{h} & =\text { distance off the ground } \\
q_{\infty} & =\text { freestream dynamic pressure } \\
L & =\text { lift } \\
\text { LatF } & =\text { lateral (side) force } \\
M_{\infty} & =\text { freestream Mach number } \\
p & =\text { static pressure } \\
p_{0} & =\text { stagnation pressure } \\
t & =\text { time } \\
T & =\text { thrust } \\
\gamma & =\text { ratio of specific heat capacities } \\
\mathrm{CG} & =\text { centre of gravity } \\
\text { config } & =\text { vehicle configuration } \\
\mathrm{EJ} 200 & =\text { EuroJet } 200 \\
\mathrm{GPS} & =\text { global positioning system } \\
\mathrm{HLLC} & =\text { Harten-Lax-van Leer Contact } \\
\mathrm{HST} & =\text { High Speed Testing } \\
\mathrm{JST} & =\text { Jameson-Schmidt-Turkel } \\
k-\omega & =\text { k-omega turbulence model } \\
\mathrm{LSR} & =\text { Land Speed Record } \\
\mathrm{LST} & =\text { Low Speed Testing } \\
\mathrm{SA} & =\text { Spalart-Allmaras } \\
\mathrm{SSC} & =\text { SuperSonic Car } \\
\mathrm{SANS} & \text { Reynolds-Averaged Navier-Stokes }
\end{array}
$$

\section{Introduction, Background and Motivation}

\section{A. The Bloodhound Land Speed Record Project}

$\mathrm{T}$

HE Bloodhound Land Speed Record (LSR) project was established in 2007 by Richard Noble and Andy Green with the engineering objective of extending the absolute World Land Speed Record to 1,000 mph from its current value of $763 \mathrm{mph}$ (Mach 1.02) [1]. Based on experience from preceding LSR projects [2, 3], it was clear from the outset 


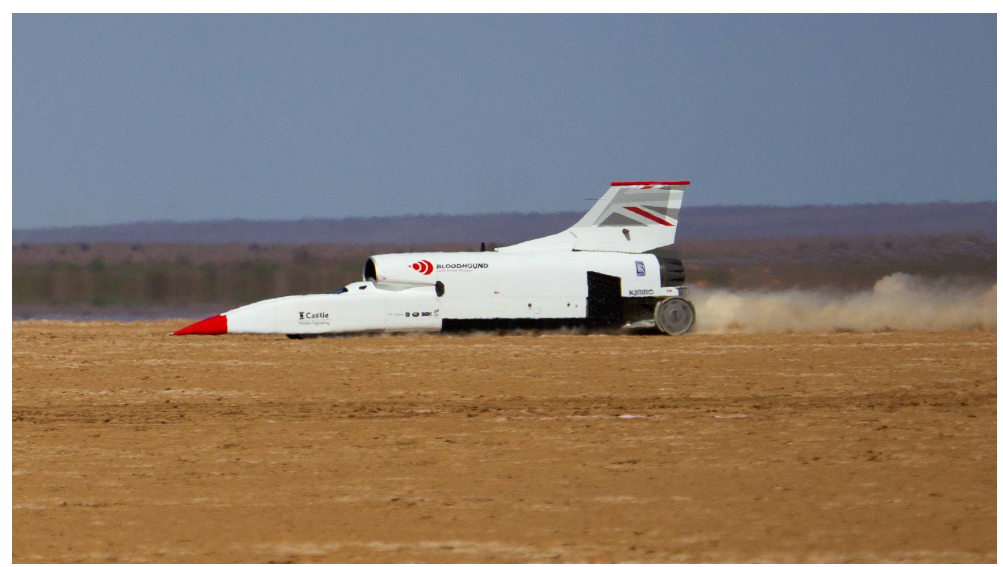

Fig. 1 The Bloodhound LSR car during HST at Hakskeen Pan, South Africa, October 2019. The presence of dust and particle entrainment into the flowfield around the vehicle is evident.

that the aerodynamic design of the vehicle would be critical to the project's success. The aerodynamic phenomona experienced by an LSR vehicle, particularly at the transonic and supersonic speeds achieved by recent LSR contenders, are complex involving transient shock systems, shock-boundary layer interaction and transonic/supersonic ground effect. All of this is complicated by the fact that a significant volume of particles is entrained into the flow off the surface. For these reasons, the Bloodhound LSR vehicle has been regarded as an excellent test-bed to understand the capabilities of modern CFD approaches to the modelling and prediction of complex aerodynamic phenomena. The High Speed Testing (HST) programme for Bloodhound, conducted on the Hakskeen Pan, Northern Cape, South Africa over a six week period in October and November 2019 was the first opportunity for the engineers responsible for the design of the car to truly understand the aerodynamic performance of the vehicle at high speed (up to $628 \mathrm{mph}$, Mach 0.82) and, critically, to evaluate the effectiveness of the CFD approaches that had been used to design the car [4]. An image of the Bloodhound vehicle during an HST test run in October 2019 is shown in Figure 1.

\section{B. Literature Review: high speed ground effect aerodynamics}

A wide ranging review of the research literature related to transonic and supersonic ground effect aerodynamics has been conducted by Doig [5] encompassing everything from the earliest, pioneering work by Mach et al on shock reflection theory [6] through to modern experimental methods and aerodynamic design approaches that have been used on ground-based high speed vehicles in recent decades [2-4]. In the review of Doig, the challenges and limitations inherent in the study of high speed bodies in ground effect using traditional experimental approaches involving high speed wind tunnels is immediately apparent [7-[10]. On top of the usual challenges related to high Reynolds and Mach number matching using scale models in a wind tunnel, the problem of replicating a high speed rolling ground-plane experimentally is significant. The 'symmetry approach' can be employed to replicate the effect of the ground-plane [7] but this has been shown to have significant limitations when the ratio of distance of the body off the ground-plane, $h$, 
to reference length scale of the body, $c$, is small $(h / c<5[5])$. When the symmetry method is used, alignment must be of the highest precision otherwise the flow will become distorted, leading to a poor representation of the moving ground-plane. Minor misalignments have also been seen to have serious and detrimental effects in some reported studies [11], and thus care must also be taken to perform careful measurements of any deflections in the wind tunnel with 'wind on’ [5].

An alternative approach to the study of high speed ground effect aerodynamics experimentally is via rocket sled methods [12]. In the work of Nakata et al [13, 14] using Japan's high speed test track facility it was possible to achieve speeds up to Mach 0.6, placing the studies into the compressibility regime but falling short of the critical Mach number. The vast majority of higher speed rocket sled test tracks are located at military installations (for example the Holloman High Speed Test Track [15]) and as a result experimental data from these facilities is not widely available in the research literature. The challenges associated with this approach for studying high speed ground-effect aerodynamics includes the effects of the geometry of the rails on the flowfield, the influence of high accelerations/decelerations experienced by the model as well as vibration and excitation of the rails due to shock impingement. All of these factors make sled based experimentation highly challenging (and often prohibitively expensive). Despite these challenges, rocket sled testing was used as a tool to validate the computational fluid dynamics (CFD) model developed for the design of the Thrust SSC LSR car [16-18] which was the first and only vehicle to set an official supersonic LSR. To date, this work represents the only effective synergy between experiments and CFD for a transonic ground effect vehicle available in the literature[5]. This analysis showed an impressive alignment between the rocket sled experimental measurements of surface pressure with the RANS-based CFD model predictions [2]. The outliers in the data were attributed to aerodynamic hysteresis resulting from the large $(\approx 50 G)$ accelerations experienced by the rocket sled model. For this reason the CFD data was chosen as the 'best predictor' of the aerodynamic behaviour of Thrust SSC.

A detailed review of the research literature pertaining to the aerodynamic design of race cars has been provided by Katz [19]. In this extensive work, one of very few references to land speed record cars, including comments on the significance of shock formation, is in relation to the design of the Blue Flame car [20] which, at $630 \mathrm{mph}$, held the LSR between 1970 and 1983. Extensive work has been conducted in recent decades into compressibility effects for automotive vehicles such as the works of Keogh et al [21,22]. Due to the challenges involved in studying compressibility effects for ground-based vehicles experimentally, much of the research that has been conducted in this field in recent years has been using CFD [2, 3, 23, 24]. Despite this, there are still many challenges associated with CFD modelling of high speed ground-effect vehicles. For example, treatment of the wheel-ground contact patch given that the wheels can accelerate flow into the contact patch and produce local pressure coefficient, $C_{p}$, values far in excess of 1 , even at low subsonic Mach numbers [5]. At high speed the choice of turbulence model and shock capture algorithms in RANS-based approaches can significantly effect the prediction of shock locations and shock-boundary layer interaction phenomena [4]. Also, in the case of an LSR vehicle the impact of particle (e.g. dust) entrainment into the flow becomes important 


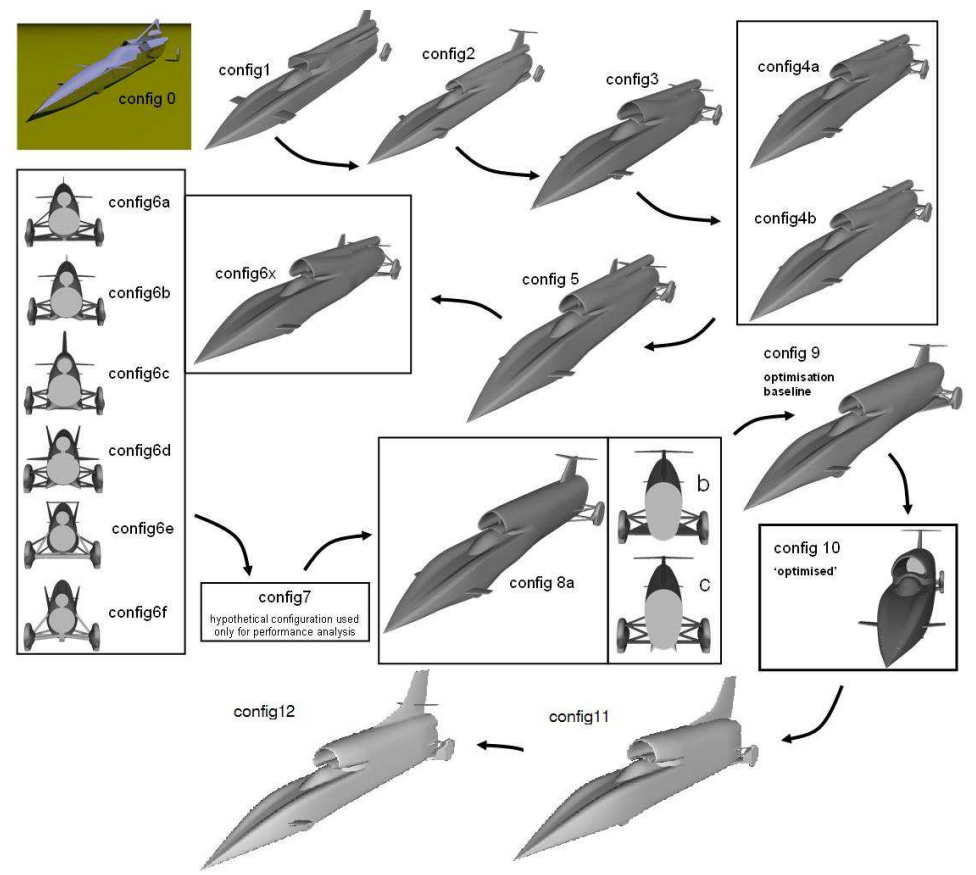

Fig. 2 Evolution of the outer mould line of Bloodhound from concept (config 0 ) through to final design (config 12) [26]

(see Figure 11. To the authors' knowledge, the only computational model developed and applied to a land-based vehicle that attempts to take into account this particle entrainment effect (or 'spray drag') is the work of Remaki et al [25] which indicates the significant drag penalty associated with this phenomenon.

\section{Design approach for Bloodhound}

Given the aforementioned challenges associated with experimental modelling of high speed ground-based vehicles, a RANS-based CFD approach was chosen as the primary tool to guide the aerodynamic design of the Bloodhound LSR car (2007 - 2015). It was deemed that this would provide the necessary fidelity of aerodynamic force coefficient predictions for optimising the design whilst keeping computational costs to a minimum. The approach used for embedding CFD into the overall design process is outlined in Evans et al [26]. The objective of the design process was to achieve a body shape that minimised the Mach-dependency of any of the aerodynamic forces or moments acting on the vehicle whilst also minimising the overall drag. Specific attention was paid to the design of the rear of the vehicle [27, 28] which was shown to be the source of much of the variations in vertical aerodynamic loads on the vehicle in the transition from subsonic to supersonic speeds [26]. The evolution of the outer mould line of the car throughout the design process is shown in Figure 2

Supersonic wind tunnel testing of the final design of Bloodhound was carried out at the Transonic Wind Tunnel Facility of the Japanese Aerospace Exploration Agency by Kleine et al [29] in 2015. The flowfield at freestream Mach numbers of 0.9, 1.1 and 1.3 was visualised using direction-indicating colour Schlieren [30]. This provided 

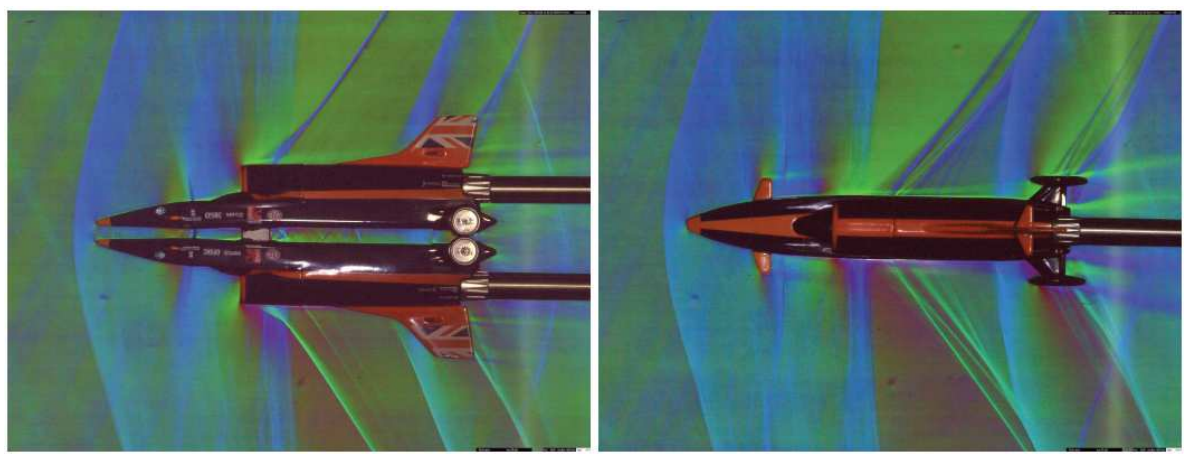

Fig. 3 Direction-indicating colour Schlieren of Bloodhound final design at a freestream Mach number of 1.1 [30]

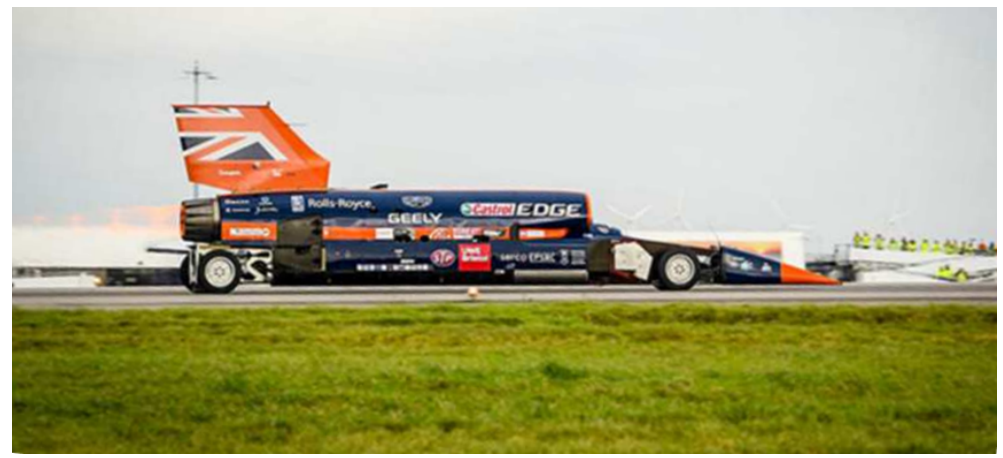

Fig. 4 The Bloodhound LSR car during LST at Newquay Cornwall Airport, October 2017

useful, qualitative validation of the RANS CFD model used during the design process but also highlighted the inherent sensitivities involved with high speed wind tunnel testing related to the accuracy of the model and model alignment using the symmetry-method approach for modelling the ground-plane. Direction-indicating colour Schlieren for the freestream Mach 1.1 case is shown in Figure 3 The asymmetry in the flowfield due to use of a low fidelity scale model and imperfect symmetric alignment is evident.

\section{Bloodhound Testing}

In October 2017 the Bloodhound vehicle was taken to Newquay Cornwall Airport (UK) to commence its testing programme. The 2017 Low Speed Testing (LST) was primarily focused on understanding the low speed and static thrust capabilities from the EJ200 jet engine system as installed on Bloodhound along with low speed (wheel) brake performance. An image of Bloodhound during a test run during the Newquay LST is shown in Figure4. The success of the LST in 2017 allowed the car to be developed and prepared for High Speed Testing (HST) in the Northern Cape of South Africa in October/November 2019. This would be the first opportunity to learn about the aerodynamic performance of the vehicle at high subsonic and transonic speeds beyond the critical Mach number.

In September 2019 Bloodhound was flown from the UK to the Northern Cape of South Africa to commence the 


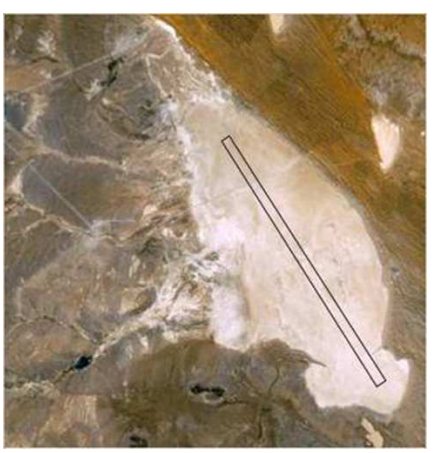

(a) Aerial view of track position within the Hakskeen Pan

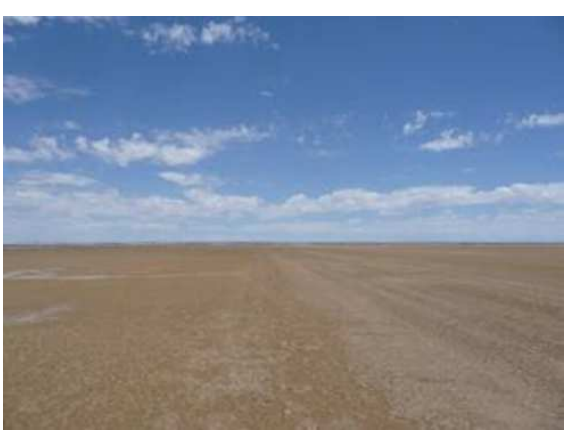

(b) Ground view of Hakskeen Pan

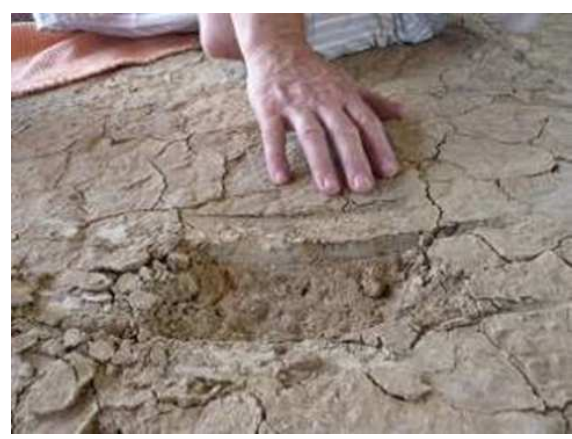

(c) Close-up of Hakskeen Pan 'alkali playa' surface

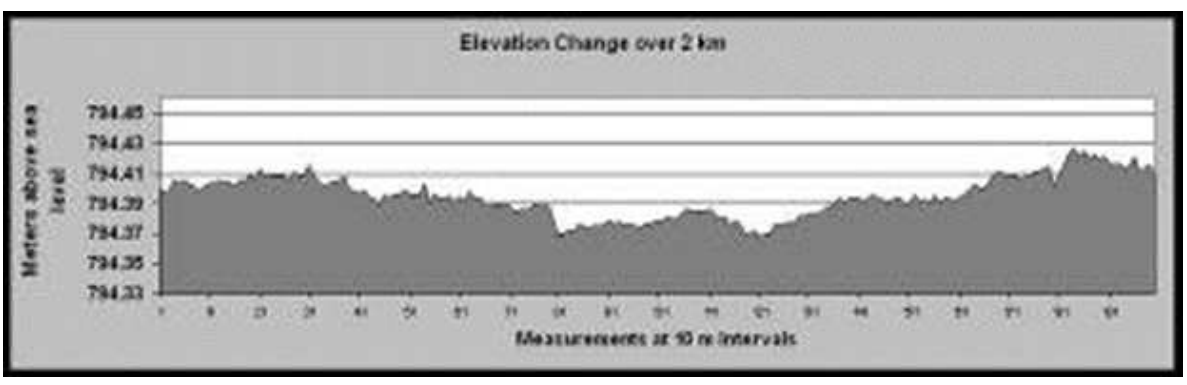

(d) Measured elevation change across a $2 \mathrm{~km}$ section of the HST track

Fig. 5 Hakskeen Pan, Northern Cape, South Africa - location for the High Speed Testing programme of Bloodhound

HST programme. The Hakskeen Pan, shown in Figure 5, had been selected as the optimal location for a 21 st Century LSR attempt due to the quality of the alkali playa surface and available space to clear and prepare a $20 \mathrm{~km} \times 1.1 \mathrm{~km}$ test track [1]. The objective of the HST programme was to generate sufficient performance data at subsonic and low transonic speeds to validate the vehicle design and accurately specify the requirements for the supplementary rocket system that would need to be installed in order to achieve a Land Speed Record of greater than $800 \mathrm{mph}$. This was the initial target before the engineering team would be able to consider higher potential speeds of up to 1,000 mph. The focus of this paper is on the aerodynamic performance data that was generated during HST and, in particular, how this data compared to CFD model predictions and influences the overall vehicle dynamic performance.

\section{E. Motivation}

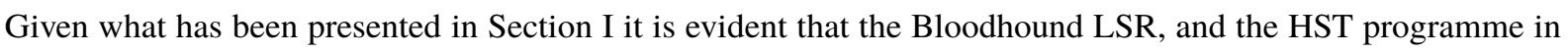
particular, provides a unique opportunity to gather uncensored aerodynamic data on a full-scale, complex-geometry, high-speed, ground-effect vehicle. The data presented in this paper can be used as a basis for a better understanding of the behaviour of such vehicles at high speed. In addition, there is the potential to improve the understanding of the capabilities of RANS-based CFD methods to accurately model flows of this level of complexity.

Despite the fact that ultimate LSR attempts are few and far between (at the time of writing the only known active 
ultimate Land Speed Record project other than Bloodhound is the 'Aussie Invader' [31]) there are a multitude of applications, identified by Doig [5], that could benefit from enhanced understanding of high-speed, ground-effect aerodynamics.

These include applications such as the proposed 'Hyperloop' transportation system in which capsules containing passengers and freight would be transported at transonic velocities in a partial vacuum tube over large distances. Experimental 'Maglev' transit systems are likely to reach Mach 0.5 in the near future [32] and the practical applications of 'Wing in Ground effect' (WIG) aircraft [33] have become popular topics of research in recent years. There is even research investigating the use of shocks generated by low-flying supersonic aircraft to suppress large forest fires [34] or suggesting that the use of a sonic boom from a low-flying supersonic jet could be used as a non-explosive weapon to injure or disorientate humans as part of a military operation [35]. It is also possible that Bloodhound HST could provide useful evidence of acceleration and deceleration effects on transonic flowfields [36] in civilian applications - a phenomenon whose study has been largely restricted to military applications (e.g. missiles) until now. Also, data on the impact of high speed particle entrainment could add important knowledge and validation datasets for those exploring the effects of particle entrainment triggered by aerodynamic phenomena such as shock systems [37].

\section{F. Paper Layout}

The remainder of this paper is structured as follows: In Section $\Pi$ the testing strategy and approach for the High Speed Testing programme of Bloodhound is outlined. This is followed by two main results and analysis sections outlining firstly the aerodynamic performance (Section IIII) of the car with comparisons to CFD model predictions and then the overall vehicle performance (Section IV]. Finally, in Section $\mathrm{V}$ a summary of the main conclusions of the HST programme along with some closing remarks is included.

\section{Testing Strategy and Approach}

The primary objective of the HST programme was not to set a new Land Speed Record but to gather sufficient data on the vehicle performance, and in particular the aerodynamic performance, to allow the engineering team to precisely specify the requirements for the additional rocket propulsion system (and any other design updates) that would be required for a Record attempt.

After some initial static testing to understand the EJ200 jet starting procedures at the 794 metre $(2,604 \mathrm{ft})$ elevation of the Hakskeen Pan and higher temperatures than experienced during LST in Newquay, UK, a series of test runs of the car was conducted at progressively increasing speeds. A summary of the full set of test runs during HST is shown in Table 1 indicating the peak speed achieved on each run, weather conditions and the objective of the run. Comments relevant to the work in this paper about the runs have been included from the more detailed driver run reports [1]. Note that the overall run number is cumulative and includes all of the test runs (numbers 1 - 21) during the LST programme. 


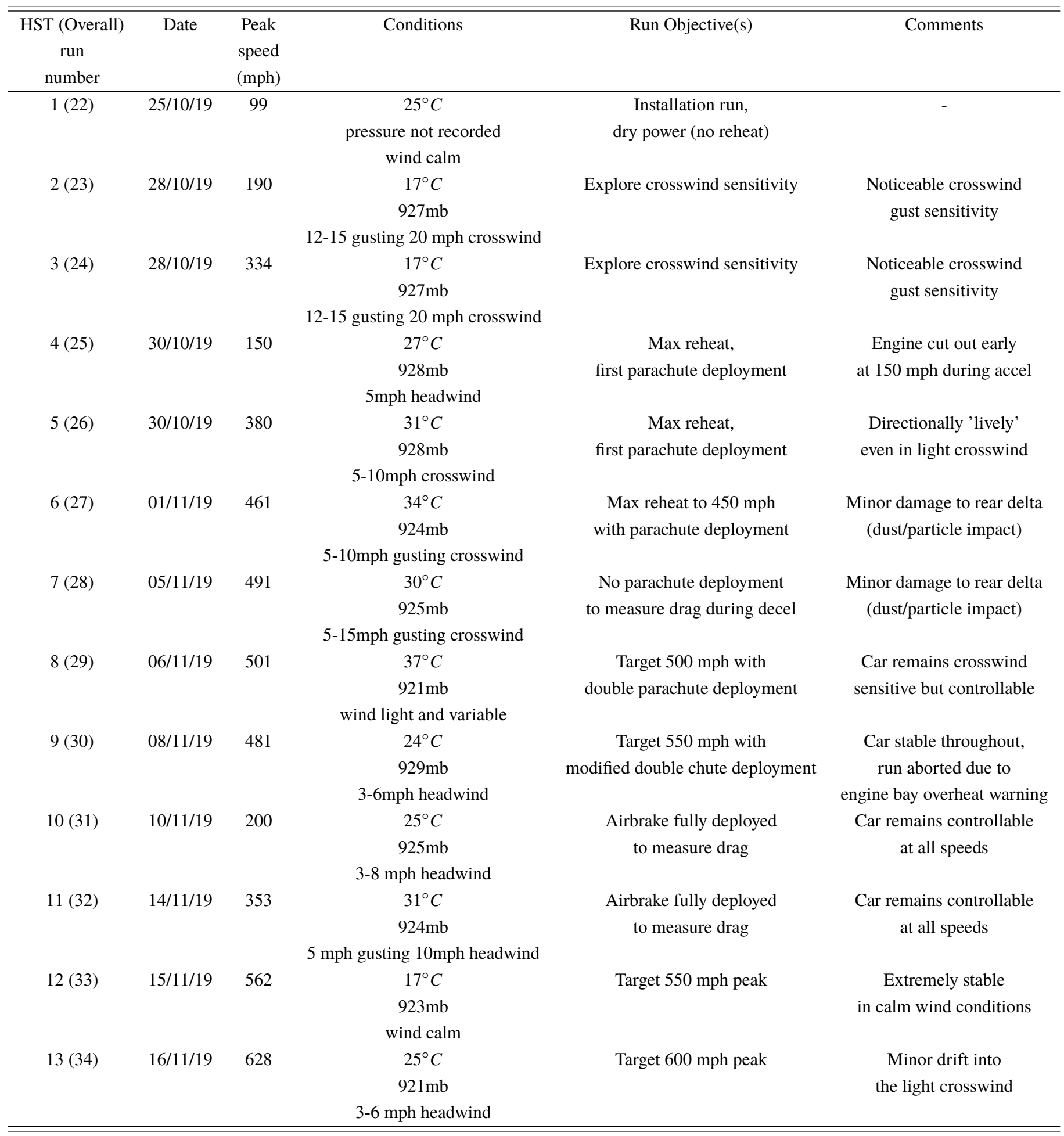

Table 1 Summary of Bloodhound HST test runs detailing atmospheric conditions, run objectives and relevant driver's comments. The overall run number (in brackets) is cumulative and includes LST runs at Newquay, UK in 2017 


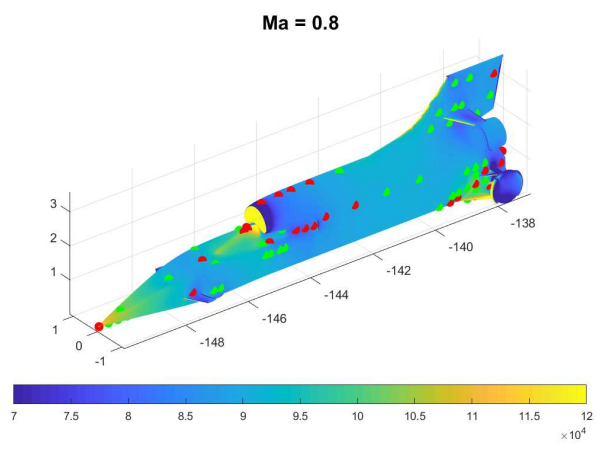

(a) View 1
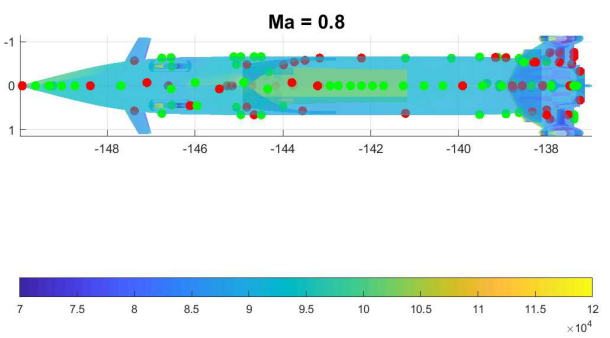

(b) View 2

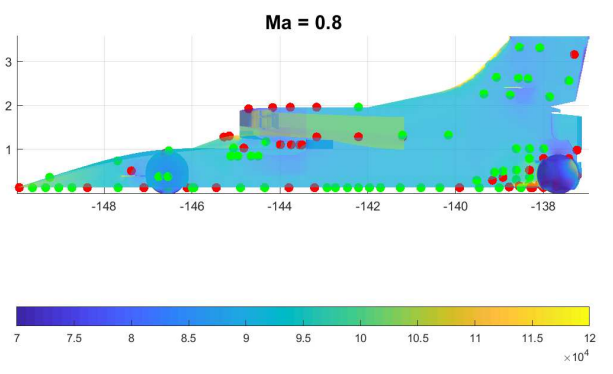

(c) View 3

Fig. 6 Positions of the 152 pressure measurement static ports over the surface of the car overlaying the HLLCSA CFD solution at Mach 0.8. Pressures are absolute in Pascals (based on an ambient pressure of 92100 Pa). In this case the red spots indicate sensors that measured pressures with an error of greater than $5 \%$ compared to the CFD prediction during the acceleration phase of HST run 13. The green spots are sensors within a $5 \%$ error tolerance.

On each of these runs, pressure measurements were made at 152 static ports located across the surface of the vehicle along with suspension deflections on each of the four wheels to allow wheel loading to be inferred. Measurements of vehicle rideheight at the front and rear of the car were made using laser sensors along with vehicle distance travelled, speed and acceleration using a combination of accelerometers and GPS. Video footage was also collected from several on-board cameras. After each run, this data was analysed to allow the engineering team to make an informed decision about whether it was deemed safe to run the car faster on the subsequent test run. Part of this analysis involved a comparison of the pressure sensor and overall wheel load measurements to pre-computed CFD predictions of the aerodynamic performance of the vehicle as a function of freestream Mach number. 


\section{Aerodynamic Performance}

\section{A. Surface Pressure Distribution}

\section{Preprocessing of HST Data}

The positions of the surface pressure sensors were chosen in order to best 'capture' the most interesting pressure distributions that were predicted to occur over the vehicle surface as indicated by the CFD simulations used to design the car. The positions of the static ports on the surface of Bloodhound are shown in Figure 6 The static ports were connected via plastic tubing to a series of digital pressure transducers located throughout the vehicle body where the pressure data was captured at a frequency of $10 \mathrm{~Hz}$. Raw data from a small random sample of these channels on HST run 13 is shown in Figure 7 (a). The first step in analysing data involved synchronising each pressure channel with the data channels recording the speed of the car as this was not done automatically. The car speed was primarily measured using GPS and this was backed up and validated via, fin mounted, pitot measurements and accelerometer measurements. The data was synchronised such that maximum/minimum pressures on each channel corresponded (in time) with the peak speed of the car. The result of this synchronisation for a selection of channels is shown in Figure 7(b).

It was observed that the there was a drift in the pressure channel data over the course of a run from the point where the pressure tranducers were zeroed (i.e. set to ambient / gauge pressure zero) through to the end of the data capture. This was attributed primarily to thermal effects which impacted each pressure channel differently depending on its routing through the engine bay from the static port on the car surfaces to the pressure transducers and recording boxes. To allow for this, each data channel was linearly scaled such that the gauge pressure measured at the start of the run (car static) and end of the run (car static) measured zero. The data was then 'clipped' to remove the pre- and post-run data. The result of this on the same selection of pressure channels is shown in Figure7/(c).

\section{Overall comparison of measured pressure distribution to CFD predictions}

During the design phase of the Bloodhound project the sensitivity of the RANS CFD predictions of lift, drag and pressure distribution across the car to the turbulence model employed and the numerical scheme within the FLITE 3D CFD software system used to design the car was studied [26]. It was found that the differences in predicted flowfield solutions were relatively minor when comparing two equation turbulence models such as k- $\omega$ [38] and SST [39] whereas the differences in flowfield solutions comparing two equation turbulence models with the simpler single-equation Spalart-Allmaras [40] were significant. In order to capture the shocks in the flowfield at transonic and supersonic speeds the HLLC convective flux scheme [4] was used whereas at lower speeds (not greatly exceeding the critical Mach number) it was possible to achieve stable solutions using the less diffusive JST scheme [41]. Steady state RANS CFD simulations were run between Mach 0.3 and Mach 1.4 in increments of 0.1 and, as a CFD validation check after each HST run, the measured pressures at each of the 152 static ports on the car were compared with the steady state CFD 


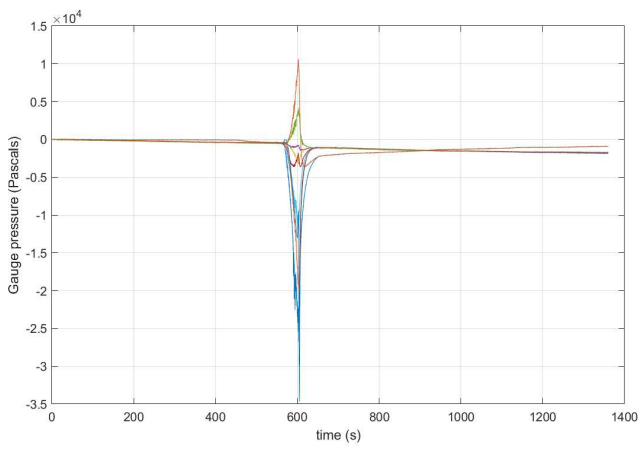

(a) Example of the raw data delivered from Bloodhound's pressure transducers

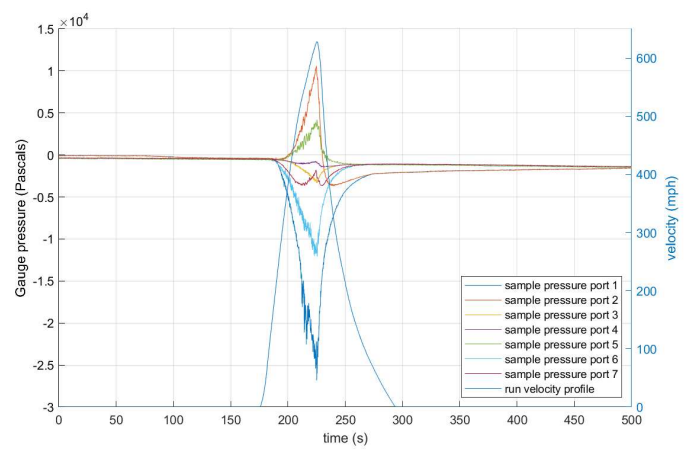

(b) Step 1: Pressure data synchronised to the velocity data

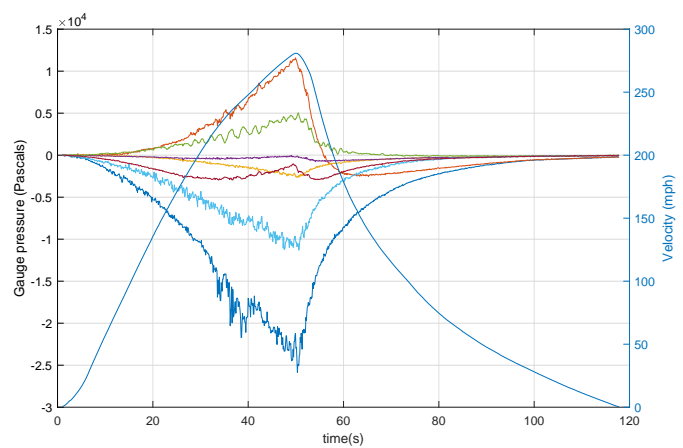

(c) Step 2: Pressure data scaled to zero gauge pressure at start and end of run and 'clipped'

Fig. 7 Example of the stages of pre-processing of a selection of pressure channel data captured during HST 


\begin{tabular}{cccc}
\hline & JST - Spalart Allmaras & HLLC - Spalart Allmaras & HLLC - SST \\
\hline \hline Mach & 0.30 .40 .50 .60 .70 .8 & 0.30 .40 .50 .60 .70 .8 & 0.30 .40 .50 .60 .70 .8 \\
\hline Acceleration (mean \% error) & 1.53 .23 .54 .15 .27 .7 & 1.61 .84 .04 .26 .08 .7 & 0.91 .52 .33 .45 .17 .3 \\
Deceleration (mean \% error) & $1.21 .82 .63 .65 .1 \mathrm{X}$ & 1.21 .92 .94 .15 .98 .8 & 1.32 .02 .53 .44 .77 .5 \\
\hline
\end{tabular}

Table 2 Mean percentage errors in CFD predictions of measured pressures across the surface of Bloodhound during HST runs 7,8,9,12 and 13. The CFD model was run with HLLC convective flux using Spalart-Allmaras and SST turbulence models and using Spalart-Allmaras turbulence model with JST and HLLC convective flux functions. The ' $\mathrm{X}$ ' in the table represents a case where a converged solution was not achieved. Note that convergence could not be achieved for any case above Mach 0.8 using the JST numerical flux.

predictions. The mean percentage error in the comparison of the absolute pressures for HST run 13 of Bloodhound during acceleration and deceleration is shown in Table 2. Given that the CFD model was based on a steady RANS approach the only difference between the accelerating and decelerating CFD simulation results is due to the boundary conditions applied at the the jet intake and jet exhaust under full throttle (acceleration) and idle (deceleration) [26].

It is evident from this initial, tabulated view of the data, which provides a crude insight into the effectiveness of the CFD modelling, that the mean error increases as the freestream speed increases for all cases. This is to be expected as the challenge involved in modelling the flow increases with speed as the significance of compressibility effects and pressure deviations from ambient increases with Mach number. This is certainly true at and above the critical Mach number when sonic flow (and shocks) first appear in the flow field. Overall, the combination of HLLC flux function and SST turbulence model appears to result in lower errors (although it must be noted that this is marginal) and therefore throughout the rest of this paper the majority of the focus will be on comparing the experimental data to the HLLC-SST CFD datasets.

Figure 8 shows the surface pressure sensor locations indicating those which were within 5\% (green) of the simulated value at that location and those which were outside this tolerance (red) at vehicle speeds between Mach 0.3 and 0.8 in increments of Mach 0.1 whilst Bloodhound was accelerating on run 13. Figure 9 shows the equivalent data during the car's deceleration.

It is evident that the higher the speed of the car the greater the number of sensors measuring absolute pressures outside of this $5 \%$ tolerance. In Table 2 it was shown that the mean error in absolute pressure ranges from about $1 \%$ at Mach 0.3 to $7 \%$ at Mach 0.8. At Mach $0.864 \%$ of sensors were within the $5 \%$ tolerance band implying that the mean error has a contribution from a relatively small number of sensors with large errors - presumably those being adversely affected by the factors previously mentioned.

As the vehicle speed increases the first locations to fall outside of the $5 \%$ error band are close to the rear delta suspension strut, on the underside of the nose and close to the jet intake. It is likely that the nose underside and rear delta errors are caused by the effects of dust/particle entrainment (not modelled in these CFD simulations) and that the intake discrepancies are related to a mismatch between the assumed intake performance (e.g. mass flow rate) and 
the actual performance at the given condition. At higher speeds the sensor locations showing the larger discrepancies tend to be clustered in regions where it was observed that there were high levels of dust entrainment/impact and also where predicted solution gradients are high. In these regions, inevitably, the sensitivity of the measured pressure to location of the sensor is highest and it is likely that there were some minor mis-matches between the locations where pressure was extracted from the simulation data and the actual position of the sensor on the vehicle (at least at the level of manufacturing/installation tolerances). It is also possible that the presence of the static port on the surface itself might be affecting the pressure locally at that position on the vehicle surface. Other reasons for mis-matches include the fact that the HST car was not fitted with front or rear winglets (deemed unnecessary until supersonic speeds) but these were included within the CFD model so the flowfield in the region of these geometric features is clearly affected by their presence in the CFD model (even though they are set to zero degrees angle of attack and not contributing to the lift distribution across the vehicle). Also, the assumption in the CFD model regarding the behaviour of the ground plane (i.e. that it is a perfectly flat, smooth and impervious surface) is likely to be a source of discrepancy between the model and reality.

Figure 10 shows the correlation between the HLLC-SST CFD predicted pressure coefficient, $C_{p}$, and the measured $C_{p}$ during the accelerating phase of run 13. Figure 11 shows the equivalent data during the deceleration phase. Given the number of sources of potential discrepancies between the CFD model and the reality during HST, outlined previously, this level of agreement is impressive. It indicates that both the RANS CFD modelling approach for the Bloodhound application over this speed range and the aerodynamic performance of the vehicle were as expected and broadly predictable. This is particularly the case when considering the net integrated effect of the simulated and observed pressure distribution on the vehicle into forces as will be seen in Section IV.

Figure 12 shows the positions of a selection of the outlier (i.e. worst CFD-measurement match) pressure sensor positions based on the acceleration phase of run 13 at Mach 0.8 just before reaching peak speed. The actual pressures measured at these port positions compared to the HLLC-SST CFD predictions are shown in Figure 13 It is evident that the majority of these error sensor positions are located in strong solution gradient regions such as in the region of the intake mouth, where slight mis-matches in position can lead to large pressure discrepancies, and also where any differences in the actual engine intake performance to the assumptions within the CFD model will be exaggerated. There are also error sensors on the leading edge of the rear suspension struts such as the delta fairing where particle/dust entrainment is a significant feature (see Section [III.D). Other sources of error are likely to be caused by flowfield interactions with the desert floor, that are not modelled correctly in the CFD (e.g. sensor port 145), and local geometric discrepancies between the CFD model and the actual car.

It should be noted that the majority of sensors do lie within the $5 \%$ tolerance band on absolute pressure prediction. A selection of sensor positions where measurements were well aligned with the HLLC-SST CFD prediction are shown in Figure 14. Again, the actual pressures measured at these positions are compared with the CFD predictions in Figure 15 


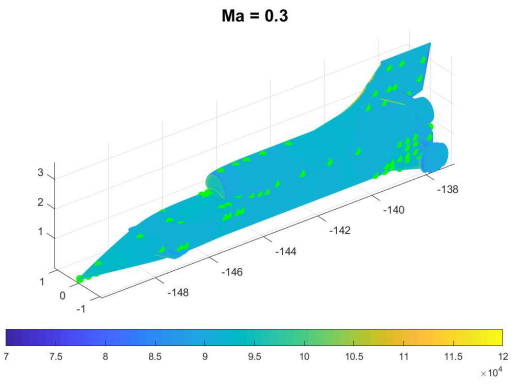

(a) Mach 0.3

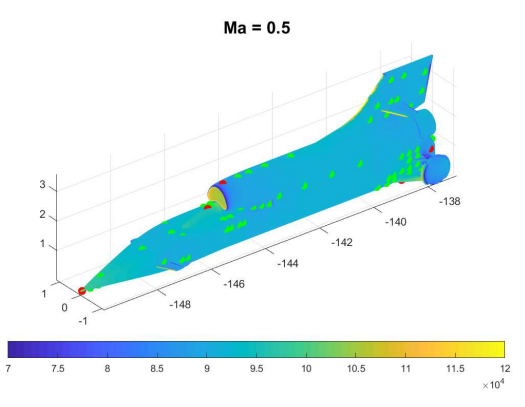

(c) Mach 0.5

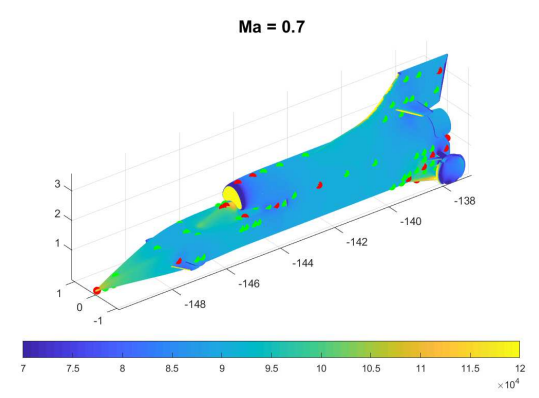

(e) Mach 0.7

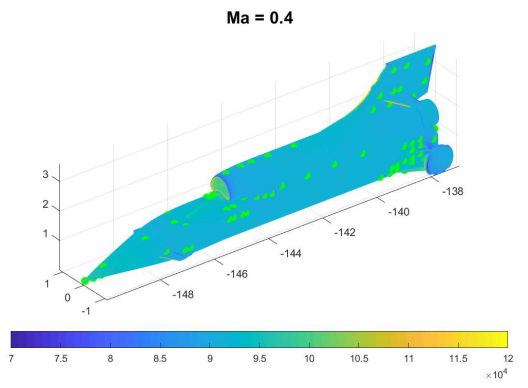

(b) Mach 0.4

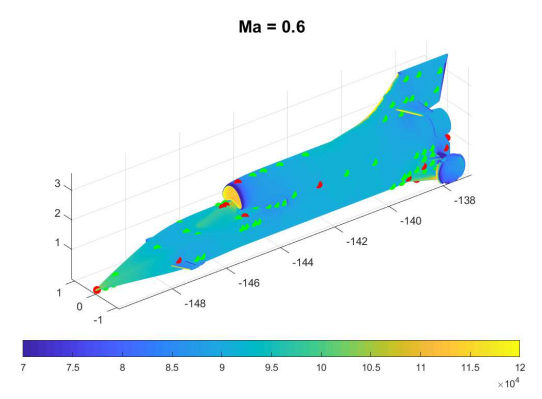

(d) Mach 0.6

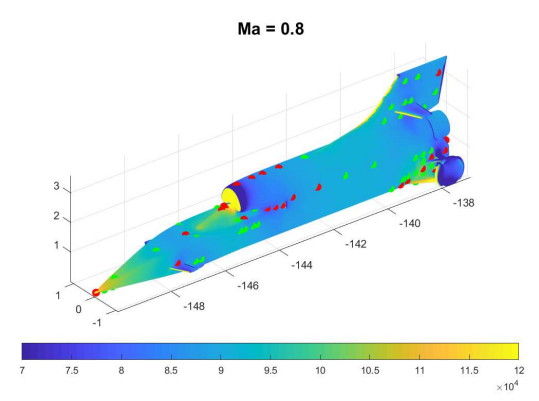

(f) Mach 0.8

Fig. 8 Acceleration phase of HST run 13: positions of pressure sensors across the surface of Bloodhound indicating sensors measuring within a $5 \%$ error band (tolerance) of HLLC-SST CFD predictions (green) and outside this tolerance (red) 


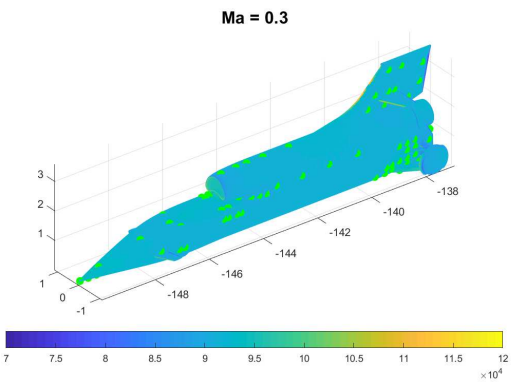

(a)

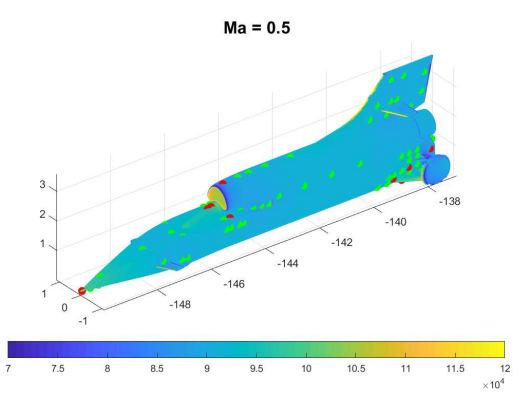

(c)

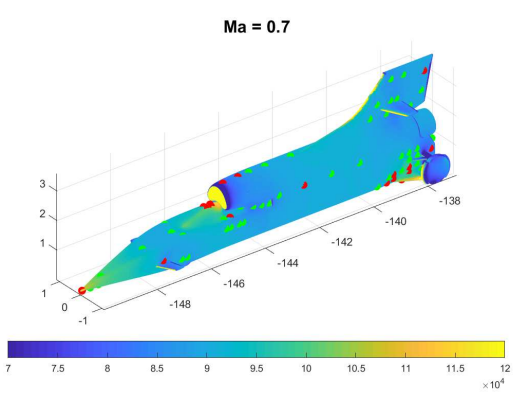

(e)

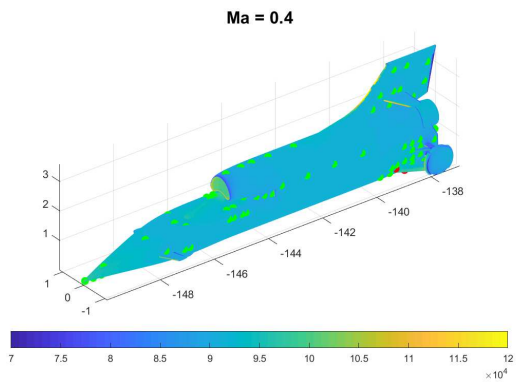

(b)

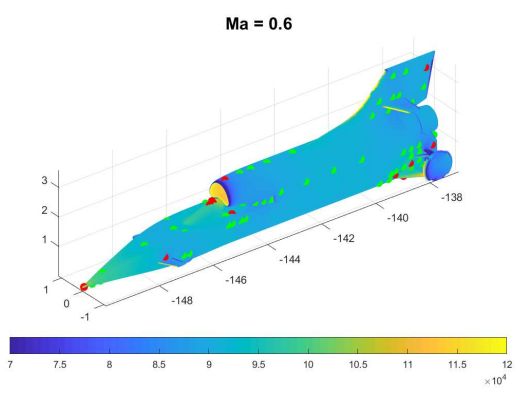

(d)

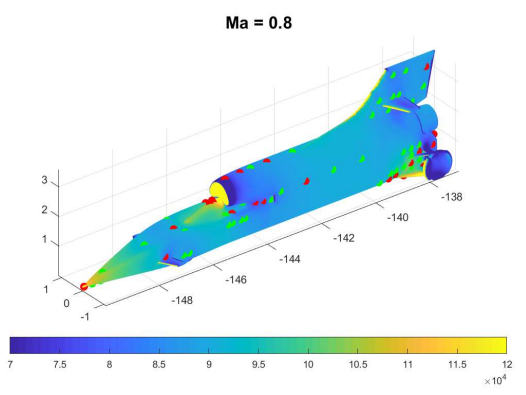

(f)

Fig. 9 Deceleration phase of HST run 13: positions of pressure sensors across the surface of Bloodhound indicating sensors measuring within a $5 \%$ error band (tolerance) of HLLC-SST CFD predictions (green) and outside this tolerance (red) 


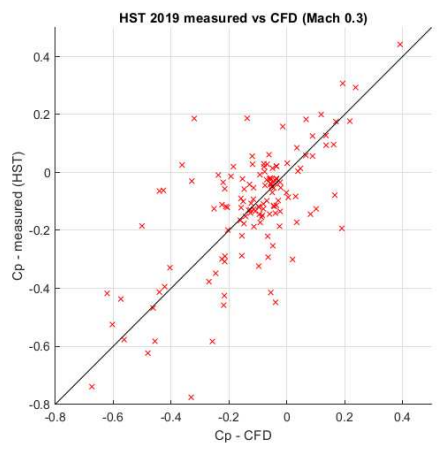

(a) Mach 0.3

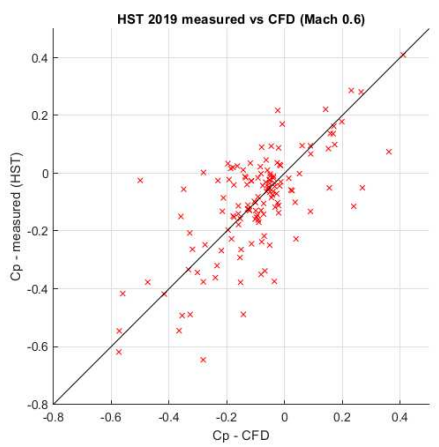

(d) Mach 0.6

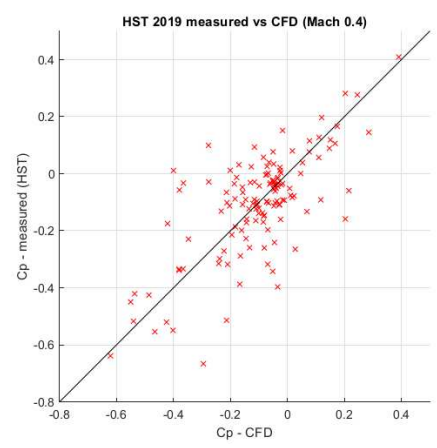

(b) Mach 0.4

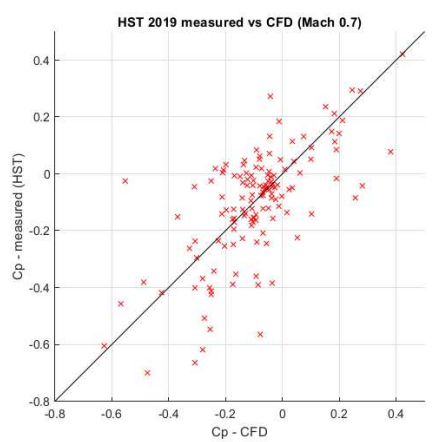

(e) Mach 0.7

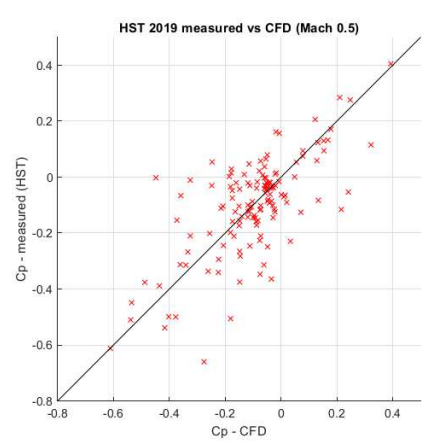

(c) Mach 0.5

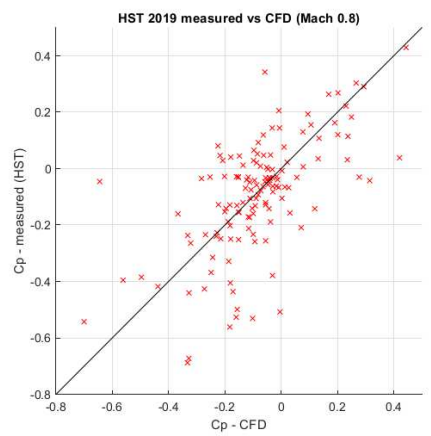

(f) Mach 0.8

Fig. 10 CFD (HLLC-SST) predicted pressure coefficients at sensor locations against measured surface pressure coefficients during the acceleration phase of HST run 13 


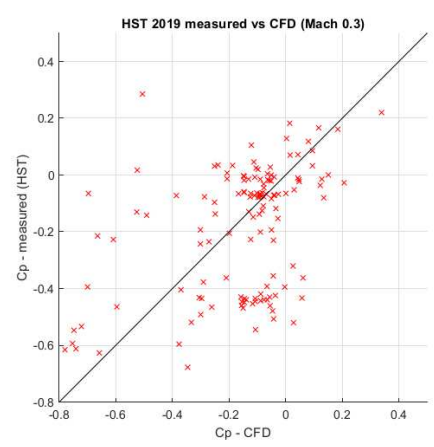

(a) Mach 0.3

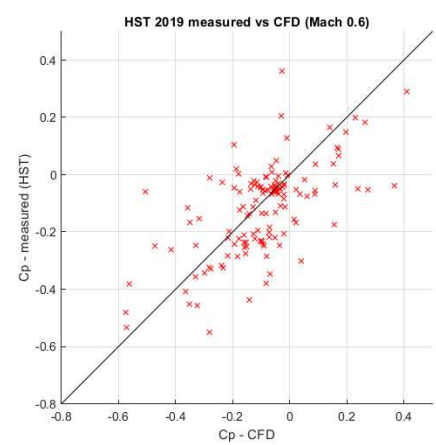

(d) Mach 0.6

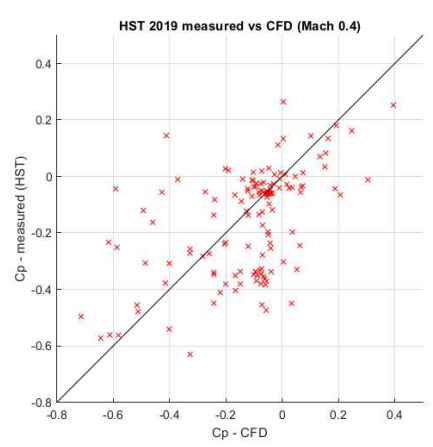

(b) Mach 0.4

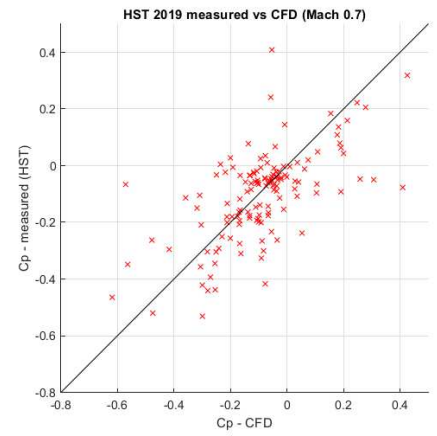

(e) Mach 0.7

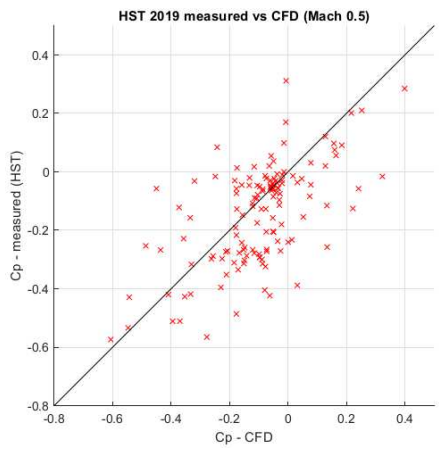

(c) Mach 0.5

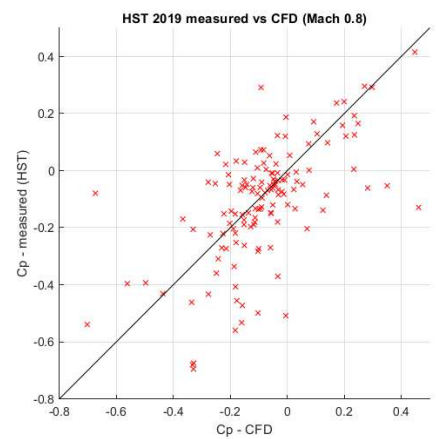

(f) Mach 0.8

Fig. 11 CFD (HLLC-SST) predicted pressure coefficients at sensor locations against surface pressure coefficients measured during the deceleration phase of run 13 (34) of Bloodhound

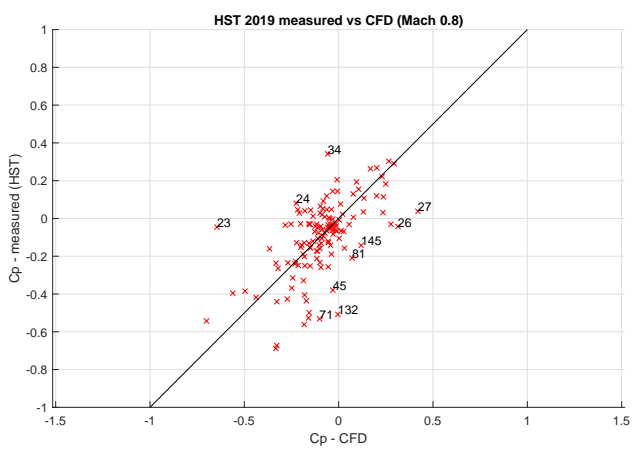

(a) CFD vs Measured $\left(C_{p}\right)$

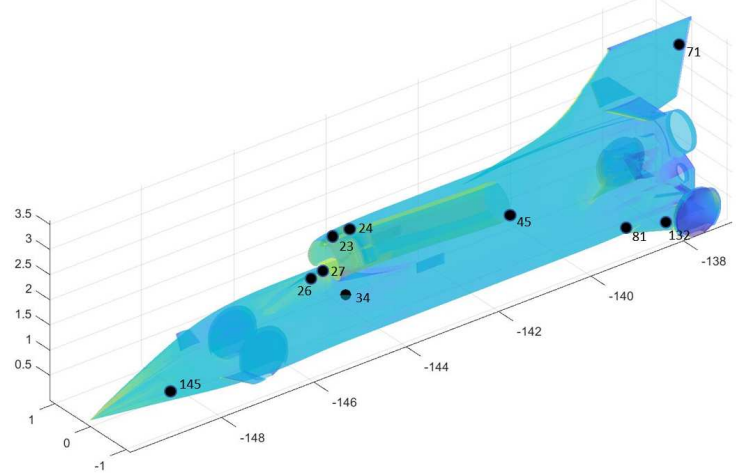

(b) Selected sensor locations

Fig. 12 Locations of a selection of the outlier sensor positions during acceleration (run 13) 

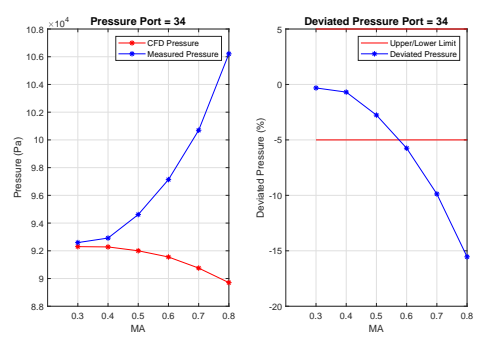

(a) port 34
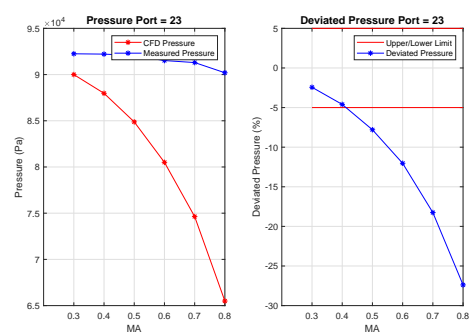

(c) port 23
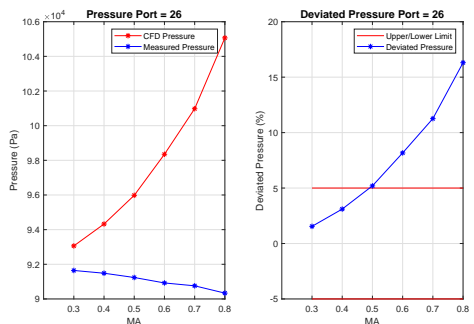

(e) port 26
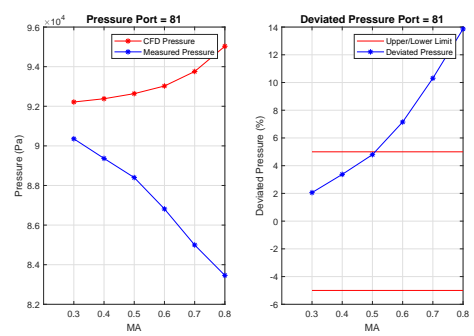

(g) port 81
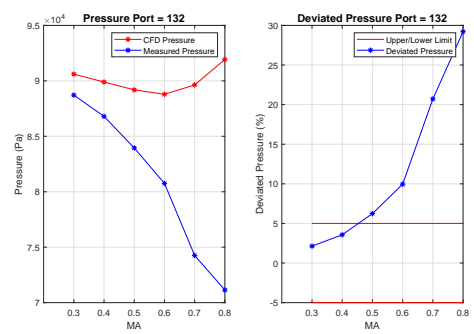

(i) port 132
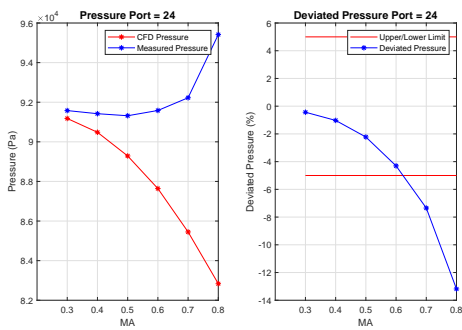

(b) port 24
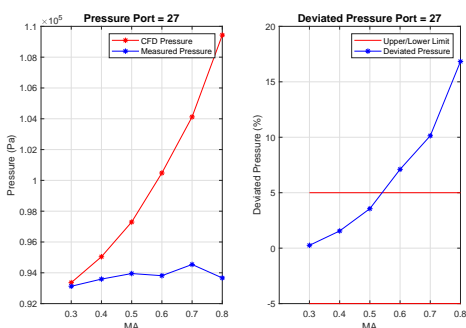

(d) port 27
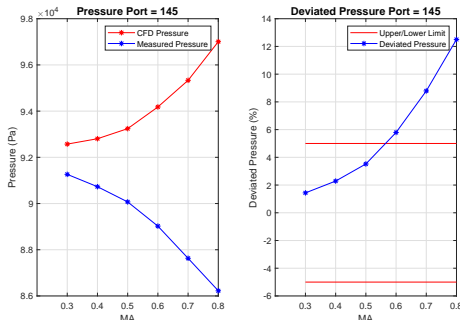

(f) port 145
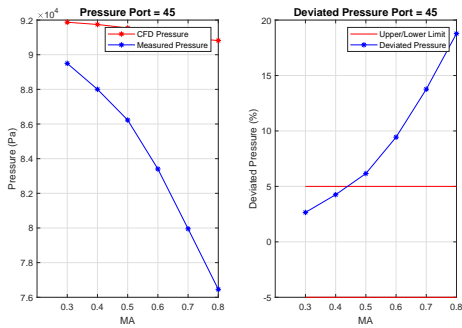

(h) port 45
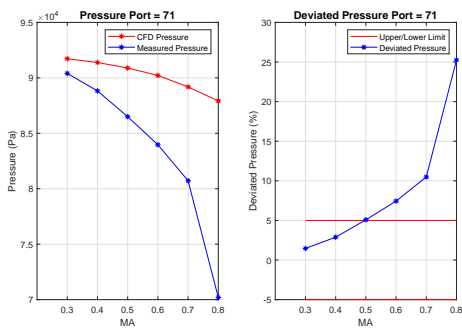

(j) port 71

Fig. 13 Measured against CFD pressures as a function of Mach number at outlier sensor (port) locations. 'Deviated Pressure' is the percentage difference between the measured pressure and HSST-SST CFD prediction indicating that all of the sensors shown measured pressures that differed by more than $5 \%$ from the CFD prediction. 


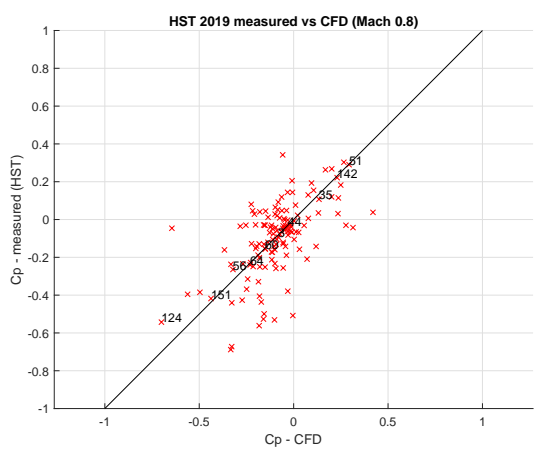

(a) CFD vs Measured $\left(C_{p}\right)$

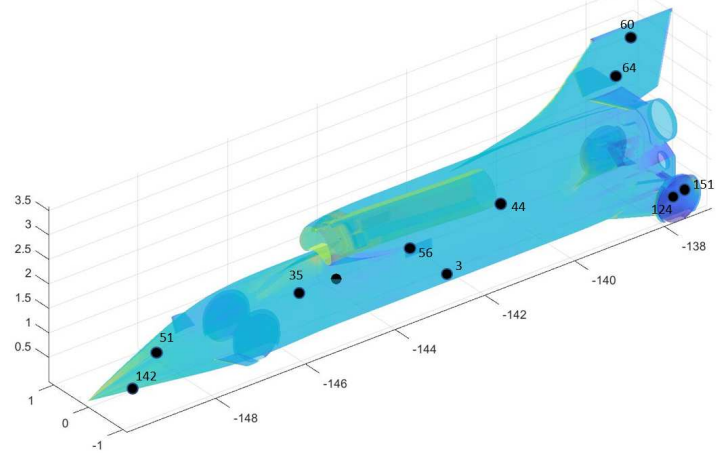

(b) Selected sensor locations

\section{Fig. 14 Locations of a selection of sensor positions showing good agreement with the CFD model during acceleration (run 13)}

\section{Centreline Pressures}

Figure 16 shows a plot of the HLLC-SST CFD pressure coefficient prediction (solid red line) versus the measured values at sensor locations on the upper centreline (blue crosses) at speeds between Mach 0.3 and Mach 0.8 during the acceleration phase of run 13. The CFD model appears to slightly overpredict the pressures on the nose at lower speeds but the correlation is excellent at vehicle speeds of Mach 0.6 and above. There is a variation between CFD prediction and measured pressures in the first couple of sensors on the centreline downstream of the intake on the upper fuselage. This could be due to a mismatch between the jet intake boundary condition applied in the CFD model, which mainly controls the mass flow rate through the intake duct, and reality and/or a local mismatch between the precise vehicle geometry and CFD model geometry. Overall, however, the CFD prediction of the trends on the upper surface of the car appears to be very good.

Figure 17 shows the pressure coefficient distribution (measured and predicted) along the underside centreline of the vehicle during the acceleration phase of run 13. Note that more sensors were positioned on the underside of the car as it was expected that the flow behaviour would be more complex underneath the car than on the upper surface. It is evident that while the CFD model does a reasonable job of predicting the overall trends, such as the nose region pressure rise and rear diffuser pressure drop (particularly at the higher speeds), there are far more discrepancies between the CFD data and measured pressures on the underside than on the upper surface. This is to be expected due to the complexity of flow physics in this region and the likely sensitivities of the aerodynamic behaviour to factors such as the vehicle rideheight and pitch. The drop in pressure predicted by the CFD model between the front wheels is not evident in the measured data but this could be due to positioning of the sensors. In future runs of the car it is recommended that additional sensors are placed in this region to try and capture this predicted aerodynamic feature. 

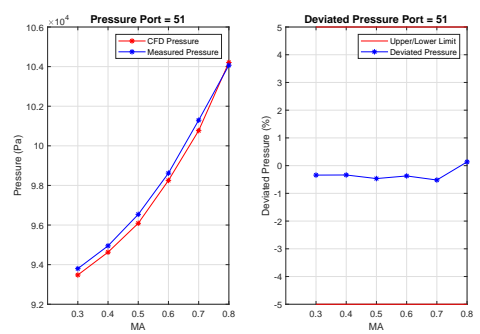

(a) port 51
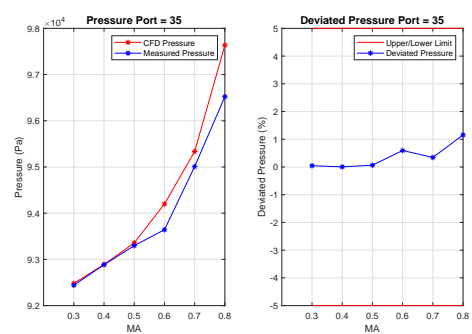

(c) port 35
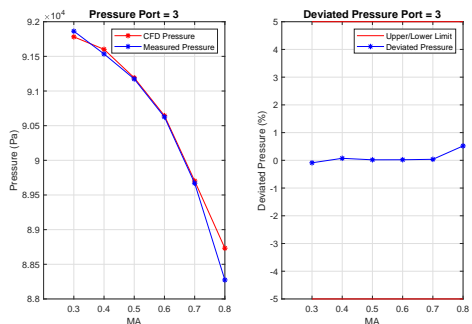

(e) port 3
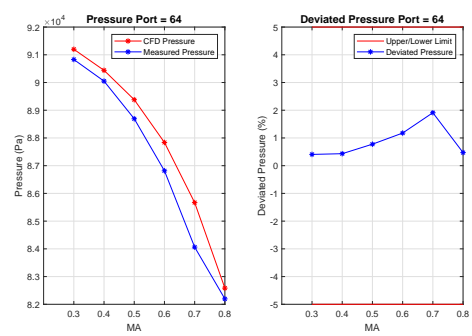

(g) port 64
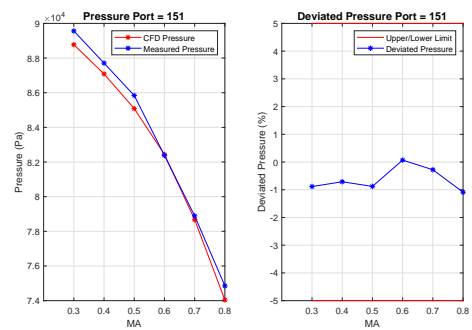

(i) port 151
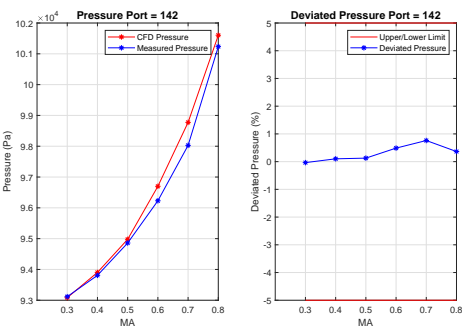

(b) port 142
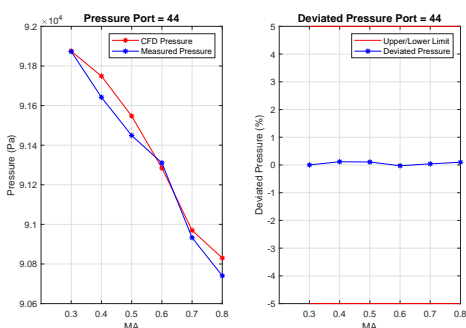

(d) port 44
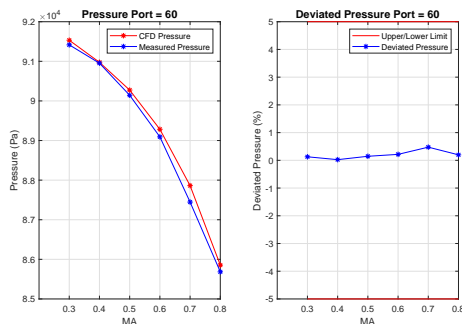

(f) port 60
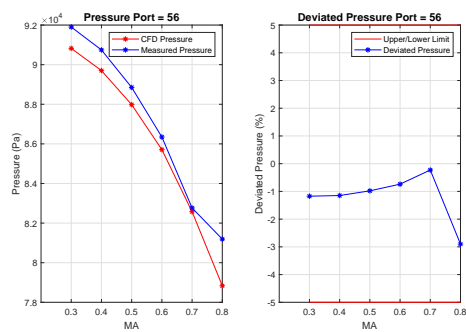

(h) port 56

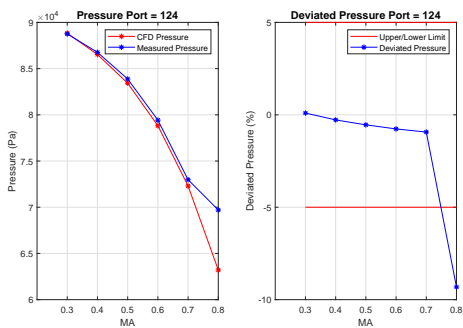

(j) port 124

Fig. 15 Measured against CFD pressures as a function of Mach number at 'good sensor' locations. 'Deviated Pressure' is the percentage difference between the measured pressure and HSST-SST CFD prediction indicating that all of the sensors shown measured pressures that deviated by significantly less than $5 \%$ from the CFD prediction. 


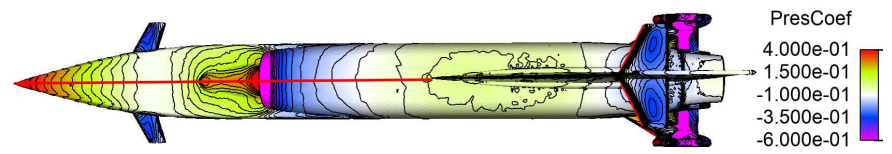

(a) Upper Cp CFD (HLLC-SST) distribution - Acceleration, Mach 0.8. Solid red line shows the centreline along which pressures were measured.

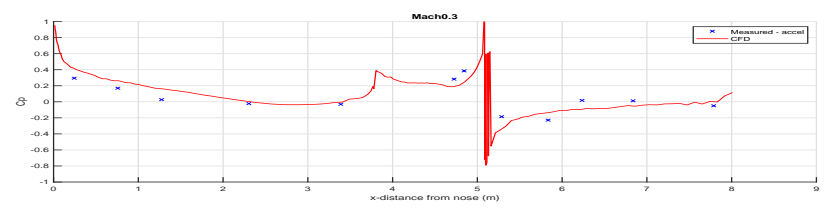

(b) Mach 0.3 (acceleration)

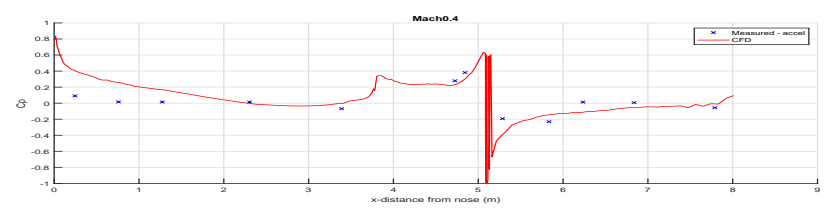

(c) Mach 0.4 (acceleration)

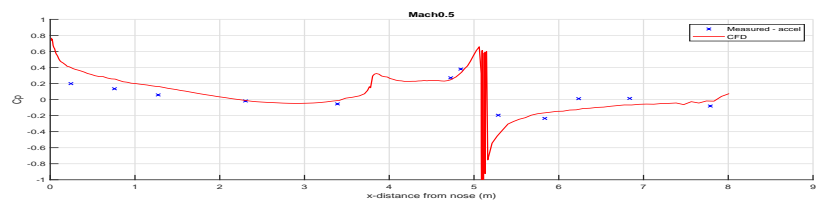

(d) Mach 0.5 (acceleration)

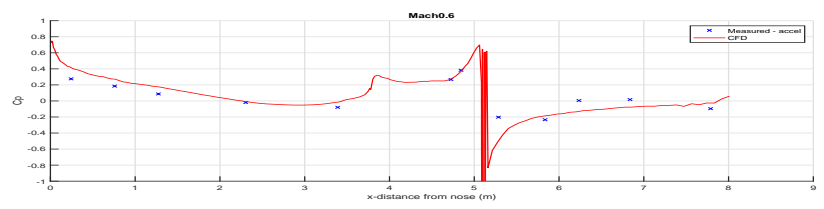

(e) Mach 0.6 (acceleration)

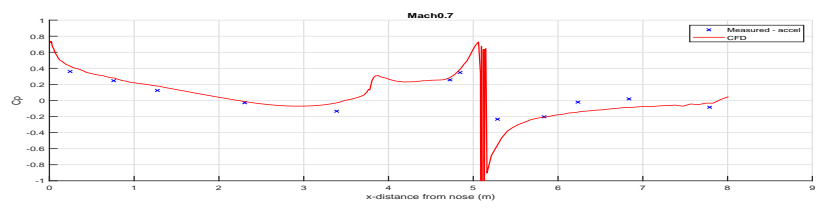

(f) Mach 0.7 (acceleration)

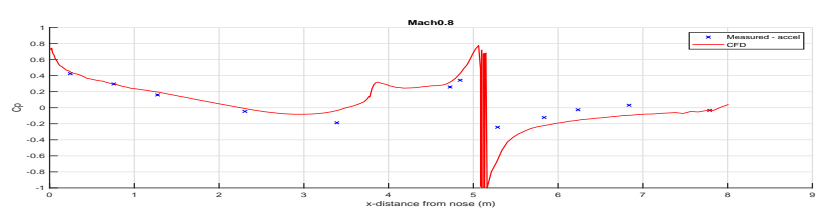

(g) Mach 0.8 (acceleration)

Fig. 16 Upper centreline pressures measured during the acceleration phase of run 13 compared with CFD predictions. Note that the discontinuity in CFD solution at $\mathrm{x}=5.1 \mathrm{~m}$ is due to the presence of the jet intake. 


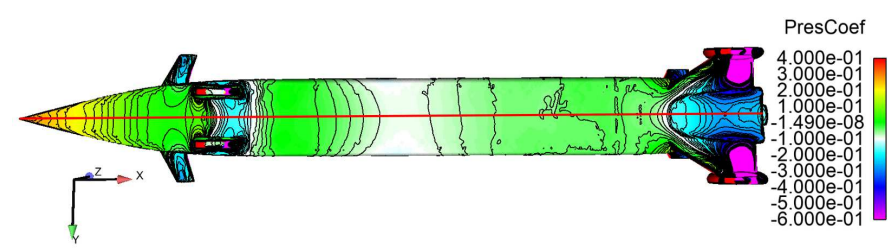

(a) Underside Cp CFD (HLLC-SST) distribution - Acceleration, Mach 0.8. Solid red line shows the centreline along which pressures were measured.

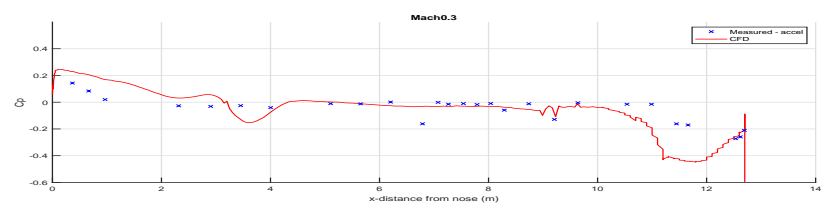

(b) Mach 0.3 (acceleration)

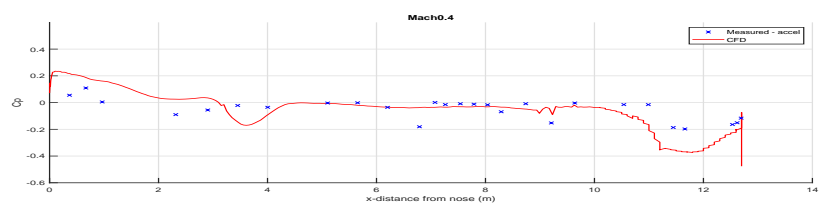

(c) Mach 0.4 (acceleration)

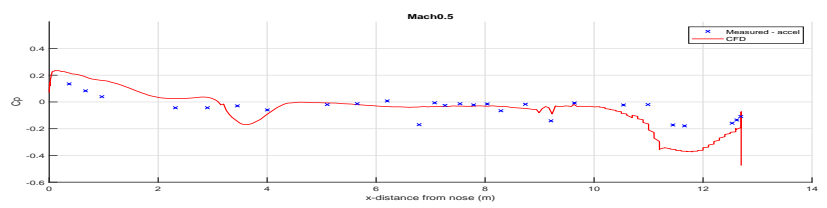

(d) Mach 0.5 (acceleration)

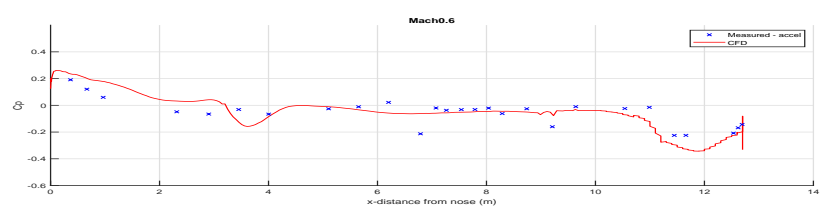

(e) Mach 0.6 (acceleration)

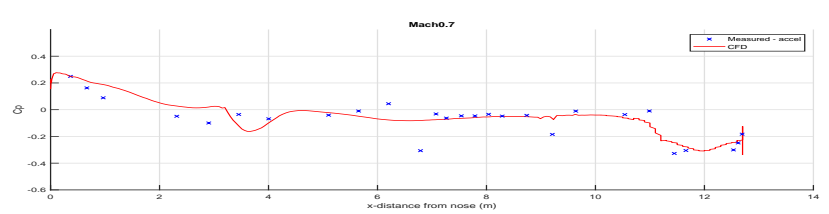

(f) Mach 0.7 (acceleration)

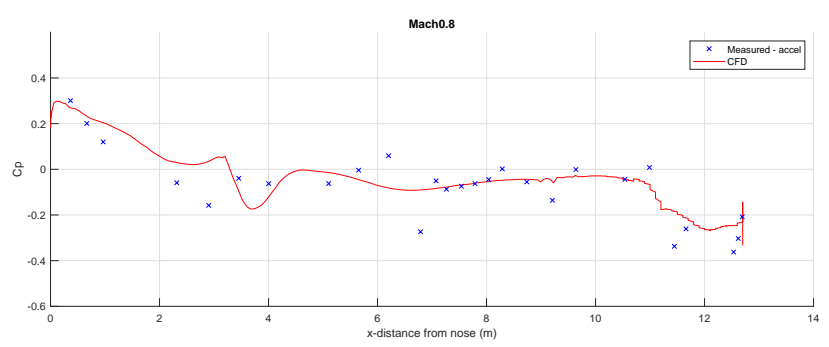

(g) Mach 0.8 (acceleration)

Fig. 17 Underside centreline pressures measured during the acceleration phase of run 13 compared with CFD predictions. 


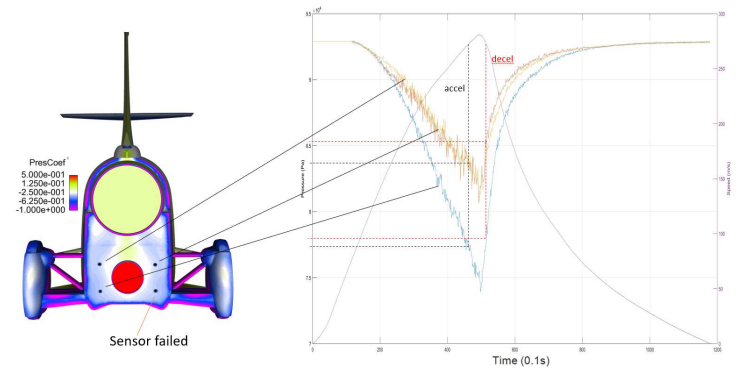

(a) Body base sensors (upper left, upper right, lower left, lower right)

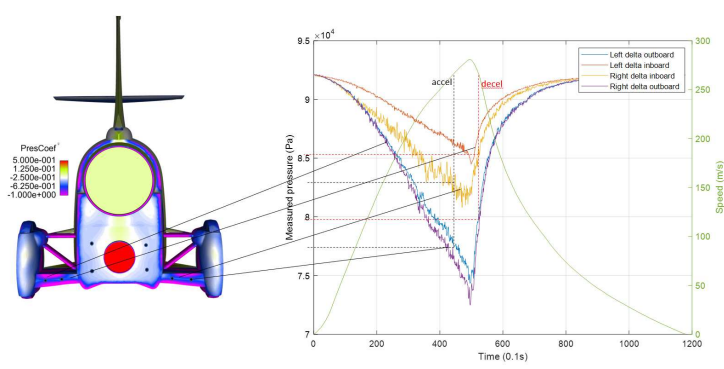

(b) Delta fairing base sensors (left outboard, right outboard, left inboard, right inboard)

\section{Fig. 18 Pressures measured on base sensors during run 13}

\section{Base Pressures and Drag Implications}

Figure 18 shows the pressures measured on 7 sensors located on the base of the car during run 13 following the scaling and synchronisation processes outlined in Section [III.A.1. Note that one of the 8 sensors positioned on the base of the car failed to record data during run 13 and is therefore missing. It should be noted that pressure traces are not perfectly symmetric (left to right) particularly in the case of the inboard delta sensors. This could be due to experimental error and/or partial blockage of the static port or tubing connecting to the transducer box. This phenomenon was not observed on lower speed runs.

A comparison of the pressures measured at Mach 0.8 during the acceleration and deceleration phases is shown. This comparison gives an indication of the effect of the jet efflux on the base pressures. Consistently the pressure measured, at a given sensor location and speed, during the acceleration phase is lower than the pressure measured during the deceleration phase. This is likely to be due to the effect of the jet efflux and inherent aerodynamic entrainment effects experienced when the EJ200 jet engine is operating in maximum thrust (reheat) mode during acceleration but throttled back to idle for deceleration. It is, perhaps, surprising to note that the effect extends as far out as the outboard delta fairing sensors which are over $2 \mathrm{~m}$ from the centreline of the jet efflux.

Table 3 shows a comparison between the accelerating and decelerating measured pressures and various CFD models used at Mach 0.8. High speed base pressures, and therefore base drag, are notoriously difficult to predict using standard RANS-based CFD methods [43]. Note that the Mach 0.8 CFD simulation under decelerating conditions (i.e. jet off) using JST flux with SA turbulence model was not stable and therefore no data is available for this case.

It can be seen that there is much variability resulting from the numerical scheme and turbulence model used in the CFD predictions. None perfectly match the measured data but, in general, the CFD tends to slightly under-predict the base pressure drop on the base of the car body (close to the jet nozzle) and significantly over-predict the pressure drop on the base of the delta fairing. This implies that, overall, it is reasonable to assume that the transonic base drag predicted by the CFD model is an over-estimate of reality. This was taken into account when developing the overall vehicle performance model based on the CFD drag predictions. The results from this model are shown in Section IV 


\begin{tabular}{ccccc}
\hline \hline & Measured - HST (Mach 0.8) & JST-SA & HLLC-SA & HLLC-SST \\
\hline \hline Base left upper (accel) & $\mathbf{- 0 . 2 6}$ & $-0.17(-36 \%)$ & $-0.29(14 \%)$ & $-0.20(-21 \%)$ \\
Base left upper (decel) & $\mathbf{- 0 . 2 7}$ & no data & $-0.18(-33 \%)$ & $-0.19(-28 \%)$ \\
\hline Base right upper (accel) & $\mathbf{- 0 . 2 6}$ & $-0.17(-35 \%)$ & $-0.27(5 \%)$ & $-0.18(-30 \%)$ \\
Base right upper (decel) & $\mathbf{- 0 . 2 6}$ & no data & $-0.18(-30 \%)$ & $-0.18(-30 \%)$ \\
\hline Base left lower (accel) & $\mathbf{- 0 . 4 3}$ & $-0.15(-66 \%)$ & $-0.37(-15 \%)$ & $-0.15(-64 \%)$ \\
Base left lower (decel) & $\mathbf{- 0 . 4 3}$ & no data & $-0.13(-70 \%)$ & $-0.15(-64 \%)$ \\
\hline Base right lower (accel) & no data & -0.145 & -0.36 & -0.15 \\
Base right lower (decel) & no data & no data & -0.15 & -0.15 \\
\hline \hline Delta left outboard (accel) & $\mathbf{- 0 . 4 1}$ & $-0.52(28 \%)$ & $-0.69(68 \%)$ & $-0.51(25 \%)$ \\
Delta left outboard (decel) & $\mathbf{- 0 . 4 2}$ & no data & $-0.62(48 \%)$ & $-0.50(18 \%)$ \\
\hline Delta right outboard (accel) & $\mathbf{- 0 . 4 3}$ & $-0.44(3 \%)$ & $-0.66(53 \%)$ & $-0.46(6 \%)$ \\
Delta right outboard (decel) & $\mathbf{- 0 . 4 6}$ & no data & $-0.58(28 \%)$ & $-0.46(0 \%)$ \\
\hline Delta left inboard (accel) & $\mathbf{- 0 . 1 8}$ & $-0.36(98 \%)$ & $-0.56(211 \%)$ & $-0.37(106 \%)$ \\
Delta left inboard (decel) & $\mathbf{- 0 . 1 8}$ & no data & $-0.48(169 \%)$ & $-0.37(106 \%)$ \\
\hline Delta right inboard (accel) & $\mathbf{- 0 . 2 4}$ & $-0.31(27 \%)$ & $-0.53(120 \%)$ & $-0.33(36 \%)$ \\
Delta right inboard (decel) & $\mathbf{- 0 . 2 3}$ & no data & $-0.46(99 \%)$ & $-0.36(55 \%)$ \\
\hline \hline
\end{tabular}

Table 3 Comparison of measured and (CFD) predicted base pressure coefficeint values at Mach 0.8 during acceleration and deceleration. Numbers in brackets indicate percentage errors in CFD predicted pressure drop compared to measured values. A negative value represents an under-estimate of the predicted pressure drop.

Figures 19 and 20 show a comparison of the measured pressures on the 7 (working) base sensors, over the range Mach 0.3 to 0.8 in 0.1 increments at which the steady state CFD models were run, to the SA and SST turbulence model results. This graphical representation reinforces the point made by the data in Table 3 that on the base of the body the CFD model does a reasonable job of predicting the pressure drop, with the SA turbulence model slightly better than SST model, and largely over-predicting the pressure drop on the base of the delta with the SA model deviating further from the measured pressures than the SST model.

Overall, it is difficult to therefore draw a clear conclusion about the best turbulence model for capturing base drag effects. But, it can be noted that that steady state RANS base drag predictions over this Mach range (0.3 to 0.8) are likely to be over-estimates for this, and similar, applications.

\section{B. Critical Mach Number}

During the design process for Bloodhound efforts were made to achieve a design with a high critical Mach number in order to delay the onset of wave drag. Largely this was achieved by attempting to keep the vehicle as slender as possible and by applying the principle of area ruling. The drag divergence Mach number is typically 1-2\% above the critical Mach number [44].

To determine a realistic estimate of the critical Mach number of the car the pressure measured at each sensor location was plotted alongside the analytical expression for pressure at Mach $1(M=1), p_{M=1}$, for the given car freestream Mach 


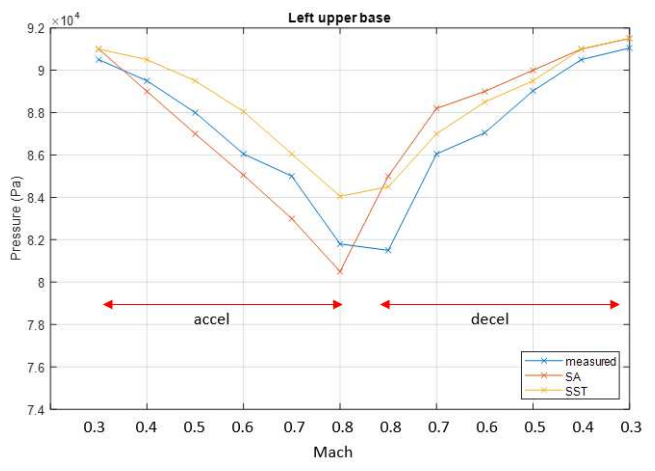

(a) Left Upper Base

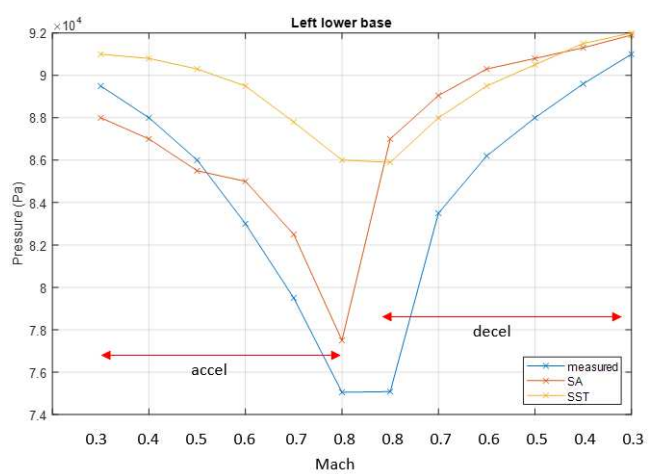

(c) Left Lower Base

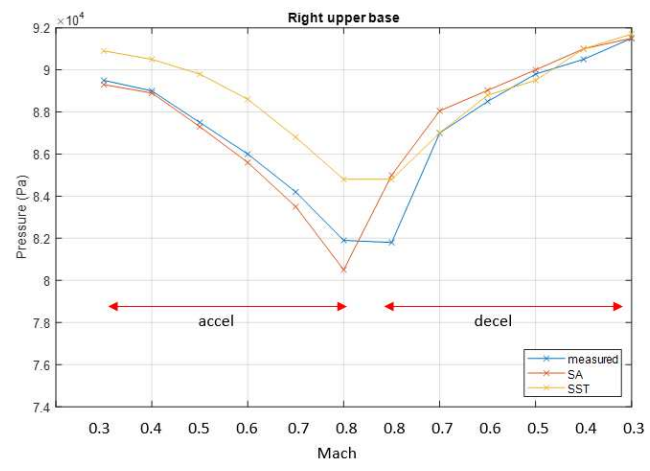

(b) Right Upper Base

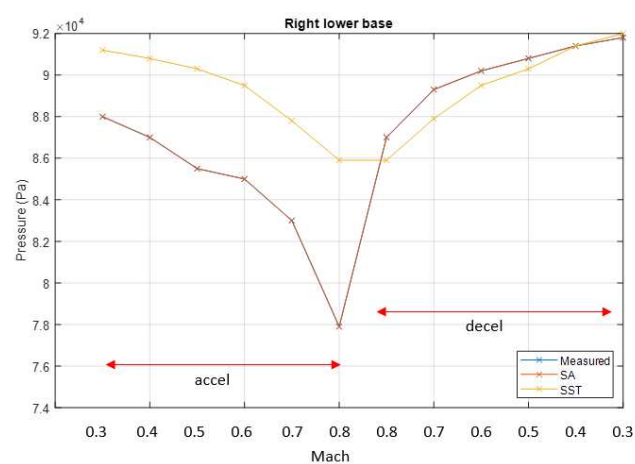

(d) Right Lower Base (N.b. no sensor data at this location)

Fig. 19 Comparison of measured and CFD predictions using Spalart-Allmaras (SA) and Shear Stress Transport (SST) turbulence models on body base 


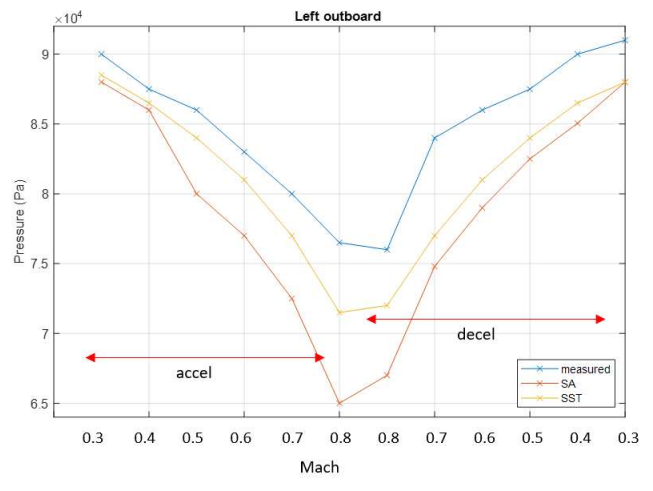

(a) Left delta base outboard

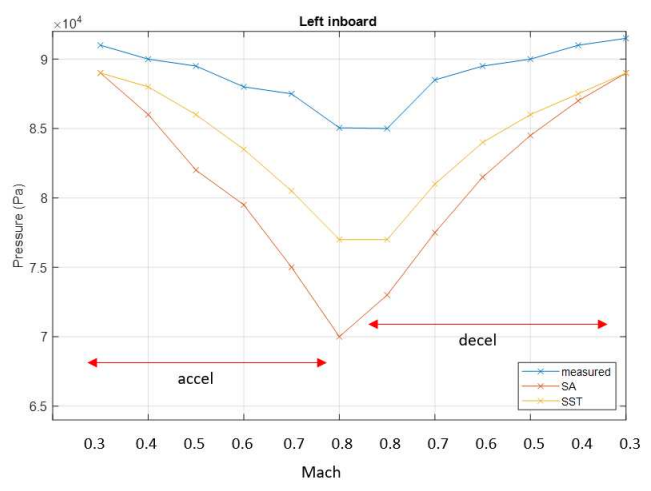

(c) Left delta base inboard

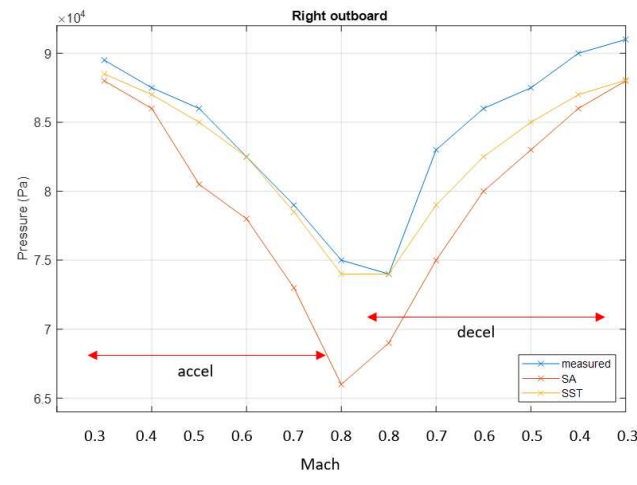

(b) Right delta base outboard

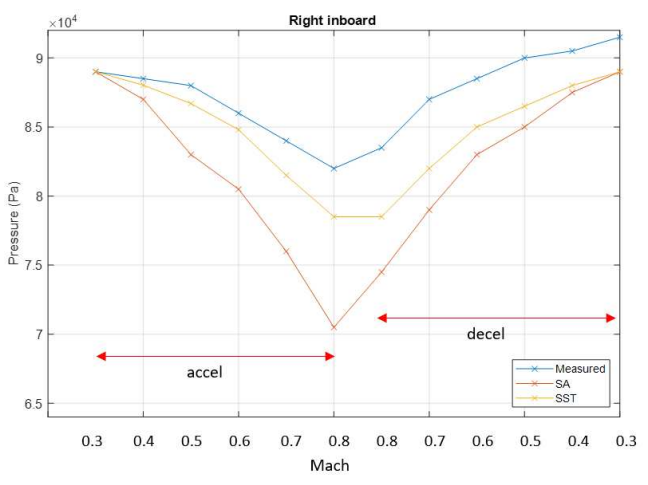

(d) Right delta base inboard

Fig. 20 Comparison of measured and CFD predictions using Spalart-Allmaras (SA) and Shear Stress Transport (SST) turbulence models on the delta fairing base 
number, $M_{\infty}$, given by,

$$
p_{M=1}=q_{\infty}\left(C_{p}\right)_{M=1}+p_{\infty}=\frac{2 q_{\infty}}{\gamma M_{\infty}^{2}}\left\{\left[\frac{\frac{1}{2}(\gamma+1)}{1+\frac{1}{2}(\gamma-1) M_{\infty}^{2}}\right]^{\gamma /(1-\gamma)}-1\right\}+p_{\infty}
$$

where $\left(C_{p}\right)_{M=1}$ is the analytical expression for sonic pressure coefficient [42], $q_{\infty}$ is the freestream dynamic pressure and $p_{\infty}$ is the freestream static pressure. Figure 21. (a) shows a selection of the sensors (in red) that indicated sonic flow during run 13 of the car. Figure 21 (b) shows the pressure measured, as well as the critical pressure curve, equation (1), for the first sensor to experience sonic flow and therefore remained sonic for longest. This sensor was located on the underside of the rear delta fairing. This, as expected, coincides with the predicted region of low pressure in the diffuser region. From figure 21 (b) it can be deduced that the speed of the car at the time that sonic flow was recorded at this location was $565 \mathrm{mph}(253 \mathrm{~m} / \mathrm{s})$ and so the critical Mach number of Bloodhound is in the region of Mach 0.73 given a temperature of $25^{\circ} \mathrm{C}$ and therefore speed of sound, $c=346 \mathrm{~m} / \mathrm{s}$ on run 13 . This is in line with the CFD predictions which indicate that the drag divergence (or drag rise) Mach number is in the region of Mach 0.75 [26].

\section{Directional Stability and Fin Pressures}

The directional (yaw) static margin target for Bloodhound is $3-5 \%$ of wheel base $(8.9 \mathrm{~m}$ ) across all operating speeds. The position of the centre of gravity of the vehicle (determined by a range of internal packaging constraints) then led to a fin design (size, shape and location) that attempted to achieve this [26]. In practice it turned out to be impossible to keep the (CFD) predicted static margin within the 3-5\% target and the predicted actual range spanned from just below $3 \%$ at low subsonic speed to near $10 \%$ at Mach 1. This meant that at higher speeds the vehicle was, although inherently directionally stable, more sensitive to crosswinds and gusts than desired. This led to the crosswind and gust operating envelope which was determined on the lower speed runs 2 - 9.

Figure 22 shows the pressures measured during run 13 (which was conducted in a light and steady crosswind) on symmetric pairs of sensors located in a matrix across the left and right hand sides of the car's vertical stabiliser (fin). For zero yaw and zero crosswind, the aerodynamic response on these sensor pairs would be expected to be identical. Although pressures measured on sensor pairs, in general, appear to be similar they are certainly not identical indicating that there is an asymmetric aerodynamic response on the fin. This is likely to be due to the light crosswind and also the car's tracking which can lead to small yaw angles as the driver responds to non-uniform environmental conditions (e.g. gusts). At high speeds, exceeding the critical Mach number, it might be that relatively small yaw angles could induce significant asymmetries due to weak shock formations on the fin. Note that by studying wheel tracks after each run it was possible to determine the approximate yaw angles experienced by the car which, although not exceeding more than $2-3^{\circ}$, were sufficient to account for significant asymmetries in the fin response which result in the fin's restoring forces and vehicle directional stability. 


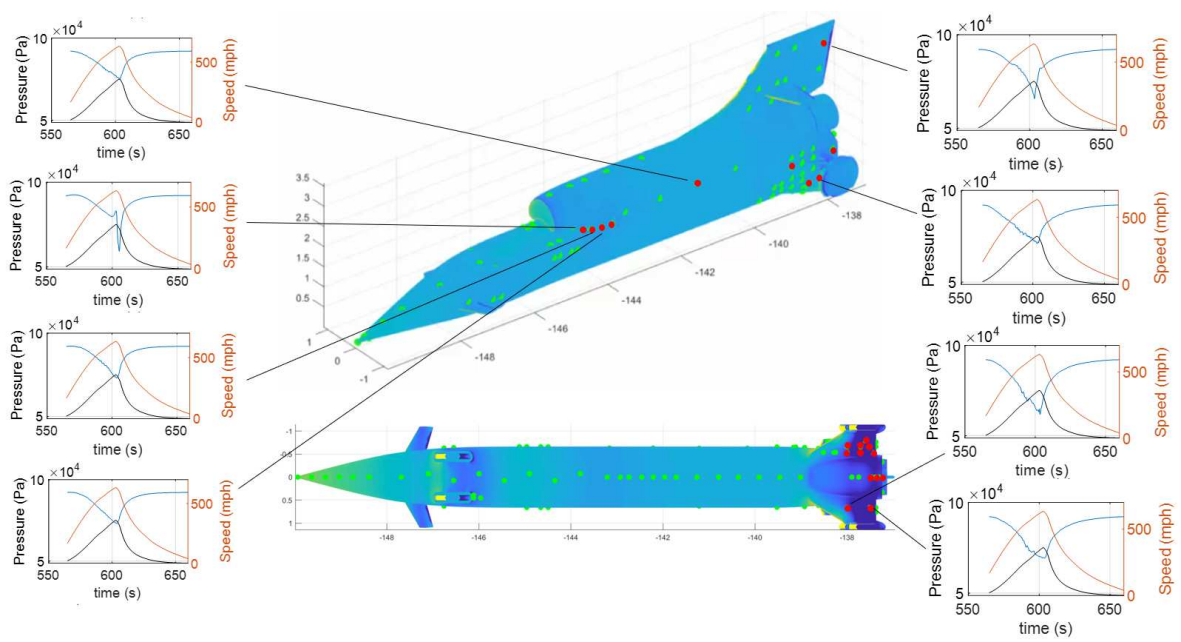

(a) Sensor locations (shown in red) experiencing sonic velocity during run 13 . Colourings on the car are mapped to CFD predictions of pressure distribution at Mach 0.8 .

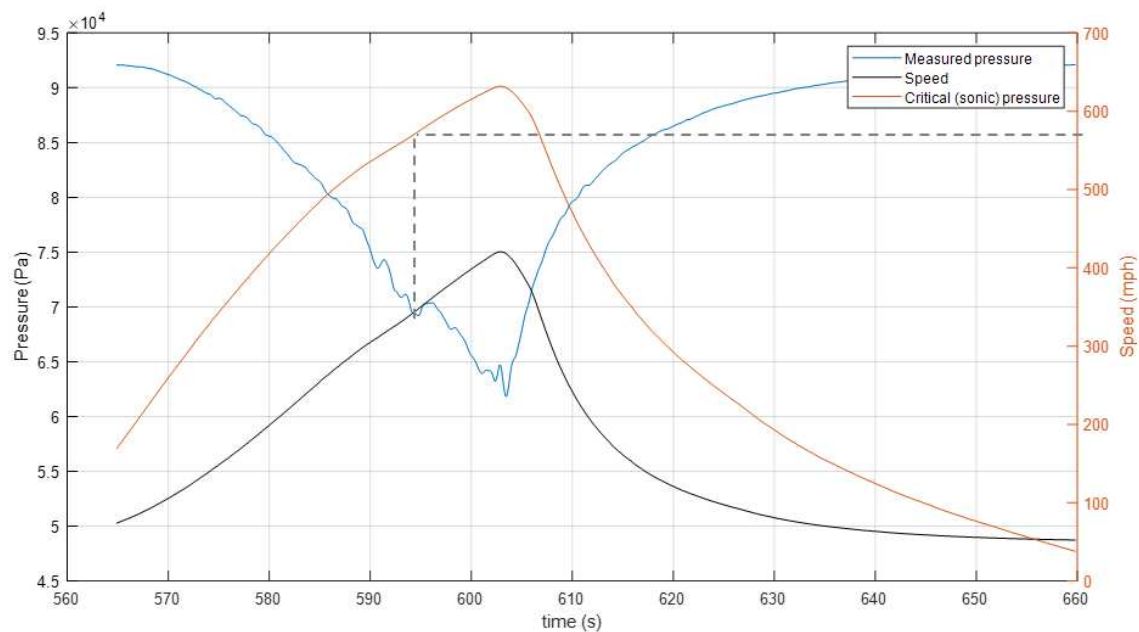

(b) Pressure measured at the first sensor to experience sonic flow (located on the underside of one of thes lower suspension 'delta' fairings)

Fig. 21 Analysis of pressure measurements at locations on the vehicle experiencing sonic flow velocities on run 13. 


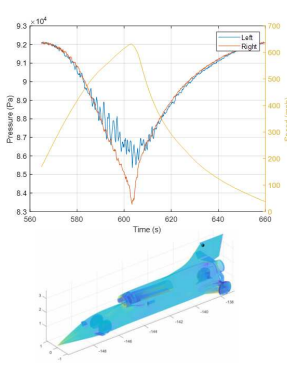

(a) Ports 59/69
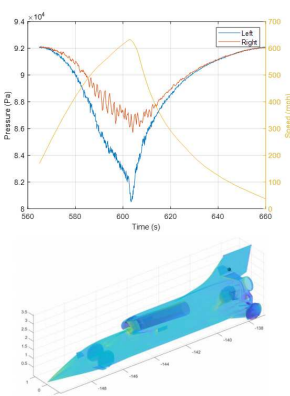

(f) Ports 64/74

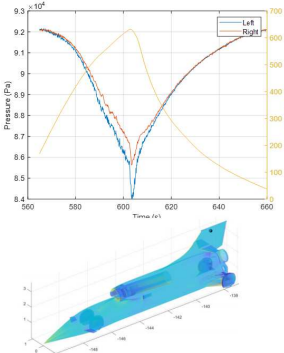

(b) Ports 60/70
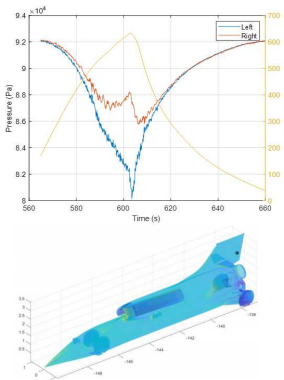

(g) Ports 65/75
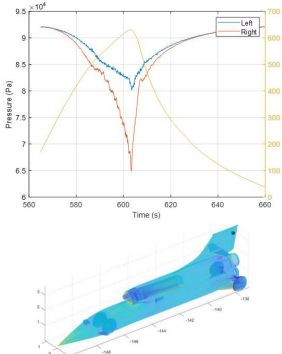

(c) Ports 61/71
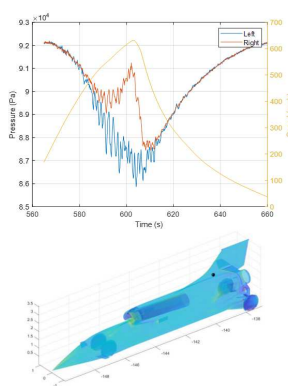

(h) Ports 66/76
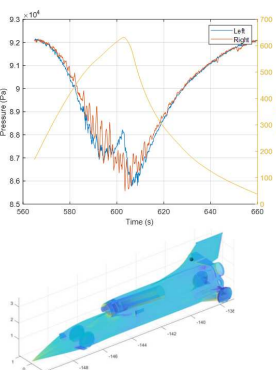

(d) Ports 62/72
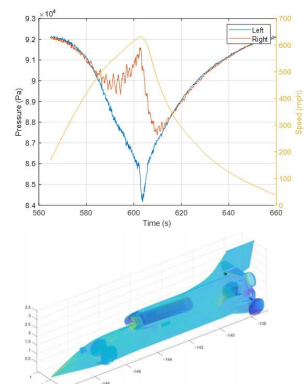

(i) Ports 67/77)

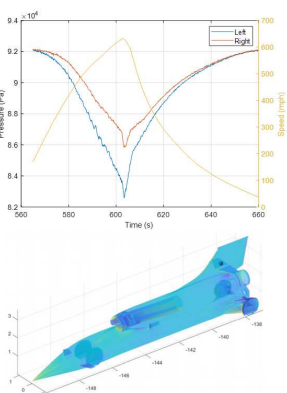

(e) Ports $63 / 73$
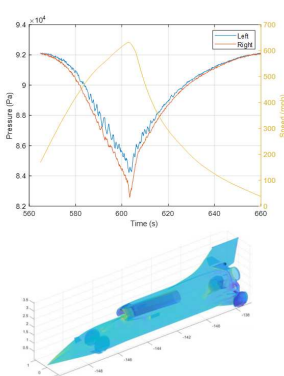

(j) Ports 68/78

Fig. 22 Pressure measurements on left hand side (LHS) and right hand side (RHS) symmetric sensor pairs during run 13 (light and steady crosswind)

Figure 23 shows equivalent fin pressure data for run 9 which, although the peak speed of $481 \mathrm{mph}$ (well below the critical Mach number), was conducted in a strong and gusting crosswind. It is possible to observe, particularly around peak speed, significant, asymmetric oscillations in the pressure readings on sensor pairs which are likely the result of the fin responses to wind gusts and driver steering inputs.

Overall, it was concluded that the directional stability, in terms of static margin, of the HST configuration of Bloodhound was sufficiently high. The large static margin in the HST configuration will allow for a reduction resulting from a rearward CG shift after fitting the rocket system for a record attempt.

\section{Particle entrainment and impact on the rear delta fairing}

From HST run 6 and beyond (i.e. $400 \mathrm{mph}+$ ) it was noticed that there was consistently damage occurring to the inboard leading edges of the delta fairing that wraps around the lower struts of the rear wheel suspension system. This is shown in Figure 24 When studying the CFD predictions of the path of hypothetical (massless) particles being entrained at the contact patches of the front wheels it became evident that the likely particle streampaths would indeed cause direct impact with the delta leading edge as seen in Figures 24 (d) and (e). Although the front wheels are $0.17 \mathrm{~m}$ inboard of the vehicle sides, the significant expansion in the flow downstream of these wheels, visible in Figure 17, causes the flow to spill from the undersides of the car. The pragmatic solution to this problem during HST was simply to 


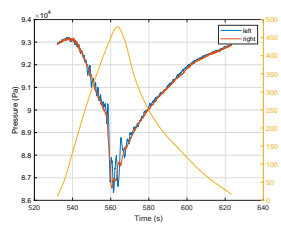

(a) Ports 59/69

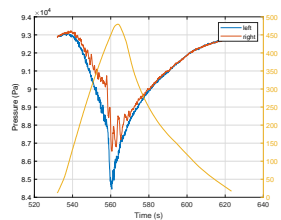

(f) Ports 64/74

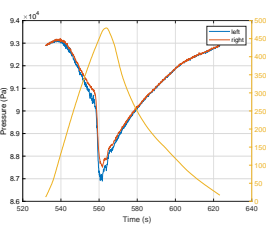

(b) Ports $60 / 70$

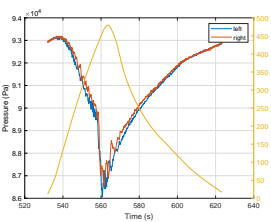

(g) Ports 65/75

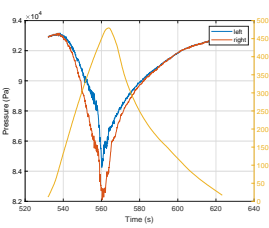

(c) Ports 61/71

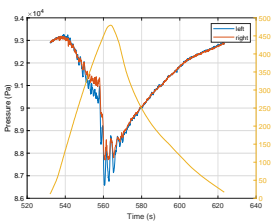

(h) Ports 66/76

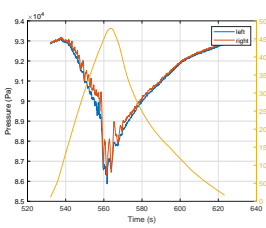

(d) Ports 62/72

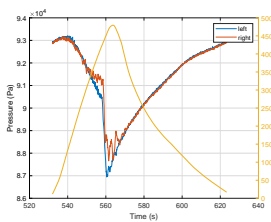

(i) Ports 67/77)

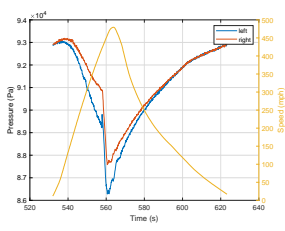

(e) Ports 63/73

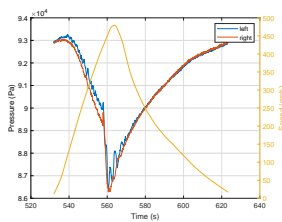

(j) Ports 68/78

Fig. 23 Pressure measurements on left hand side (LHS) and right hand side (RHS) symmetric sensor pairs during run 9 (strong and gusting crosswind)

strengthen the leading edges of the delta fairings for higher speed runs. At higher speeds there was still evidence of high momentum particle impact in this region but the damage no longer occurred. In Figure 24(c) pathlines of the flow over the rear delta upper surface and car side have been visualised due to the entrained particles scouring the surface. When compared to CFD surface streamlines in this region (Figure 24(f) ) it can be seen that where the particle scouring is taking place the flow patterns are broadly similar to that in Figure 24 (c).

Clearly there was a drag penalty incurred due to this effect. This particle impact induced drag (or spray drag) was analysed computationally in the work of Remaki et al [25]. From this work, and previous experience of spray drag on the Thrust SSC project, an extra drag term, in addition to the CFD aerodynamic drag, was estimated and used in the overall vehicle performance model (see Section IV). It might be possible to mitigate against this spray drag penalty, and minimise vehicle damage, in future car configurations by the introduction of longitudinal strakes on the underside of the car downstream of the front wheels to minimise the spill of particles from underneath the main vehicle body.

\section{Overall Vehicle Performance}

In this final results section of the paper, the overall performance of the vehicle, influenced by the aerodynamic behaviour, is analysed. It outlines the conclusions that could be drawn from measurements of loads on the vehicle's four wheels, laser rideheight measurements that allowed vehicle pitch to be measured along with the overall dynamic response (speed, acceleration, distance as a function of time) compared to a simple, force balance, performance model.

\section{A. Wheel Load Measurements}

Axle loads were determined by measurement of the travel of the four independent suspension systems using deflection sensors [45]. A pre-computed map used to convert from suspension deflection to applied load was used as 

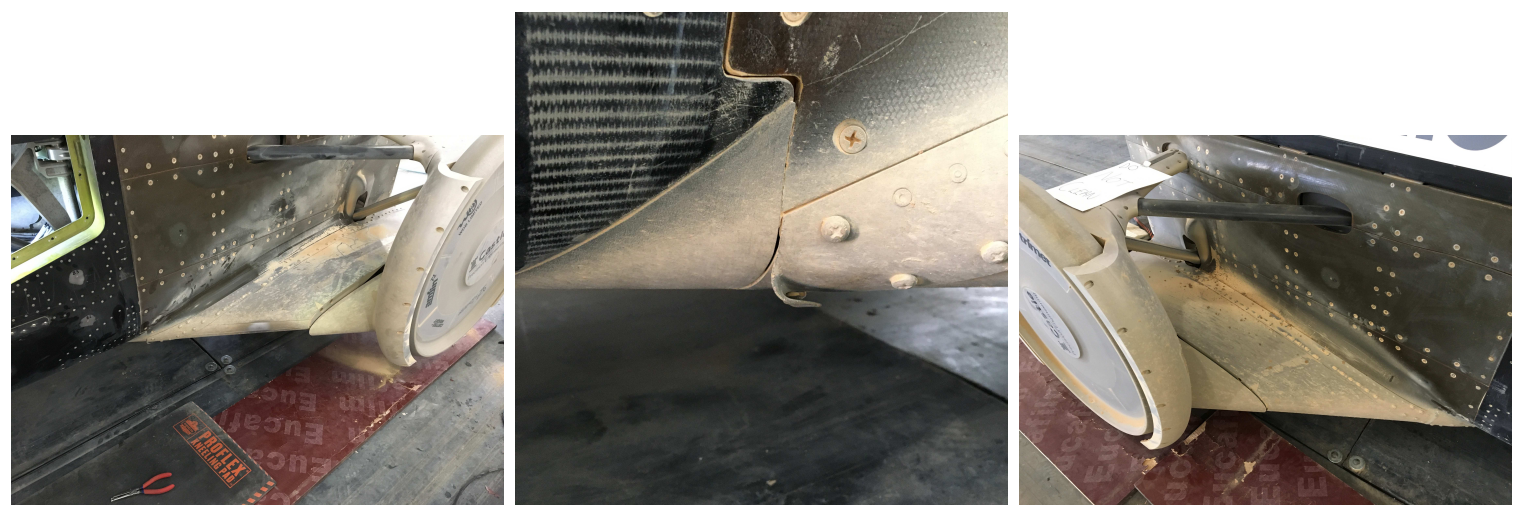

(a) Location of the rear 'delta' fairing wrap- (b) Zoom in on the inboard leading edge of (c) Evidence of 'paintwork erosion' on the ping around the lower suspension struts RHS delta fairing showing the damage caused side of the car above the rear delta to the steel skin
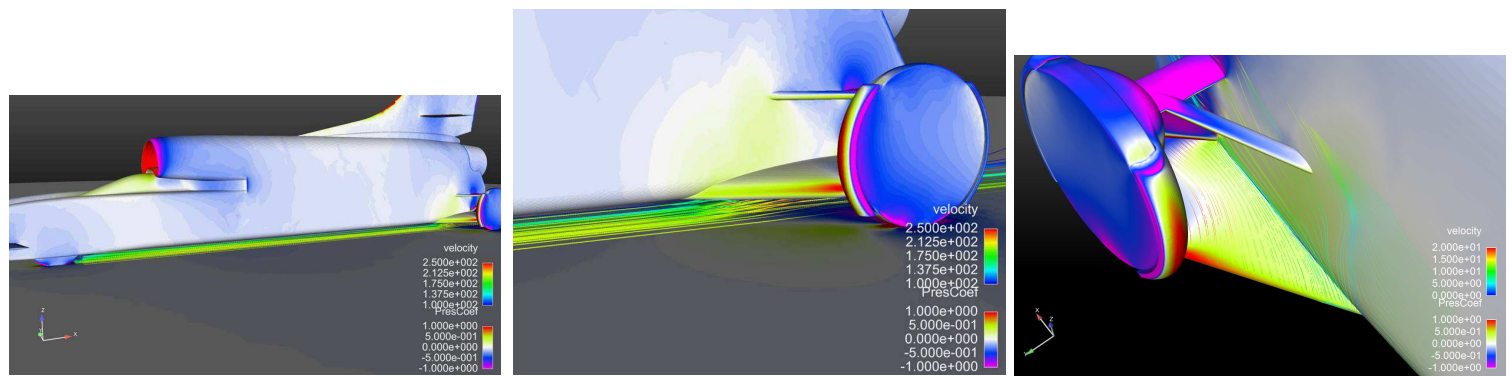

(d) CFD prediction of the streampath of par- (e) Streampath of entrained particles impact-(f) Surface constrained streamlines (coloured ticles entrained at the front wheels ing delta leading edge by 'first node off wall' velocity) in the vicinity of the right rear delta (Mach 0.8)

Fig. 24 Damage to the inboard leading edge of the delta suspension fairing on run 6 (see Table 1 


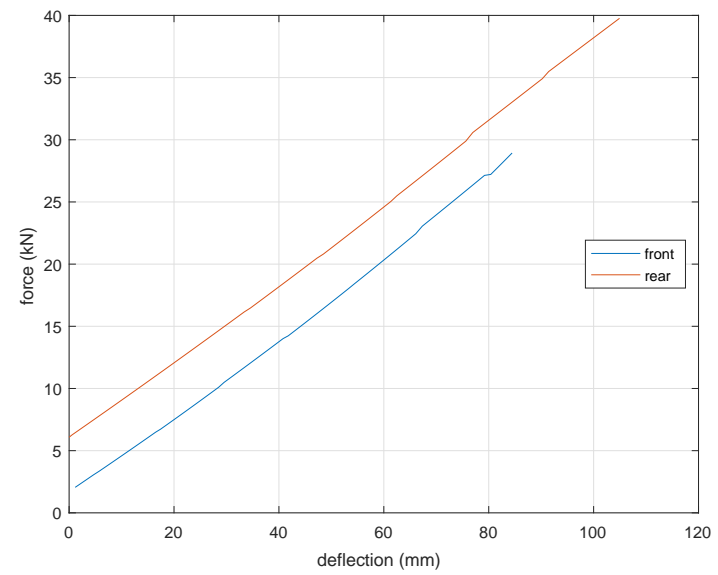

Fig. 25 Suspension deflection measurement to applied load mapping

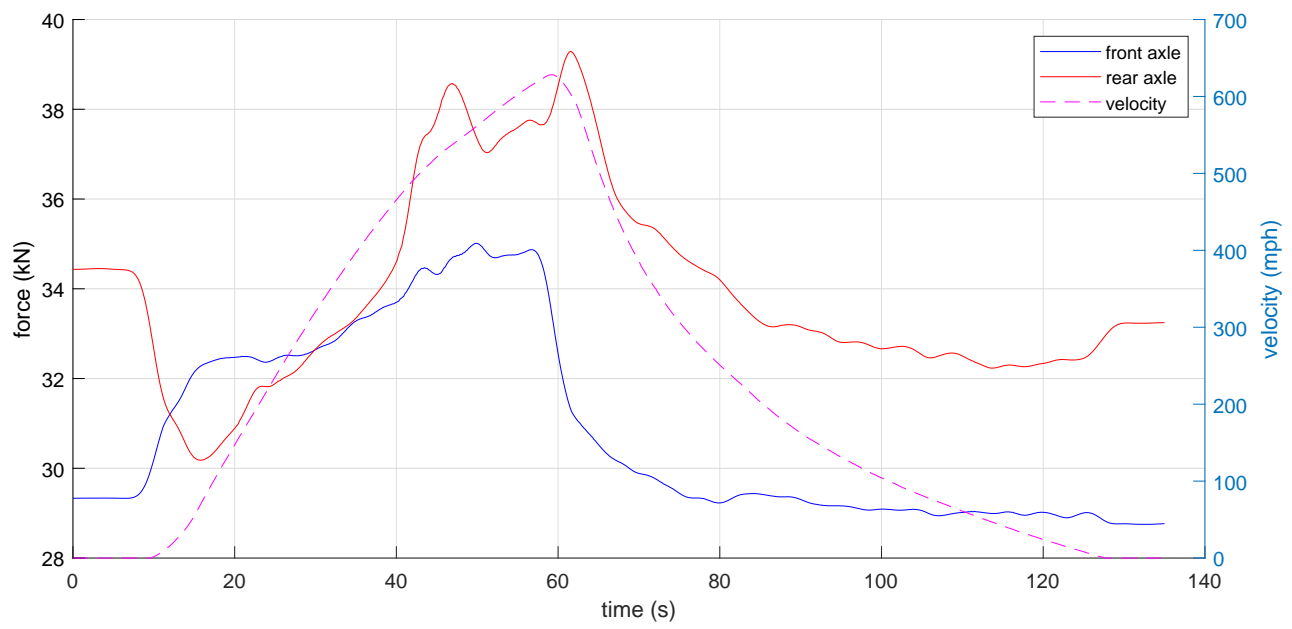

Fig. 26 Wheel loading during run 13

shown in Figure 25. This mapping was then adjusted to ensure that the start and end load distribution corresponded with the measured 46:54 front to rear bias vehicle weight distribution. The resulting front and rear wheel loading during run 13 is show in Figure 26

The factors affecting the front:rear wheel load distribution include the thrust loading from the jet as well as the aerodynamic loading. A full analysis of all these factors and how they were modelled is beyond the scope of this paper. However, it can be see in Figure 26 that there is a clear transfer of load from rear to front when the engine throttles up at approximately time, $t=8 s$. This is then followed by an increasing load (driven by increasing aerodynamic downforce) on both front and rear axles until the car reaches approximately $550 \mathrm{mph}(245 \mathrm{~m} / \mathrm{s}$ or Mach 0.71$)$ at time $=45 \mathrm{~s}$ beyond which point the rear axle load begins to drop. This is in line with both the CFD predictions [26] and the observation of critical Mach number (see Section III.B. Beyond approximately $M=0.7$ transonic effects begin resulting in the loss of 


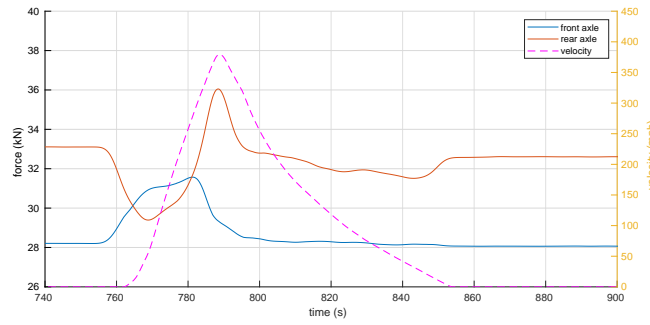

(a) Run 5

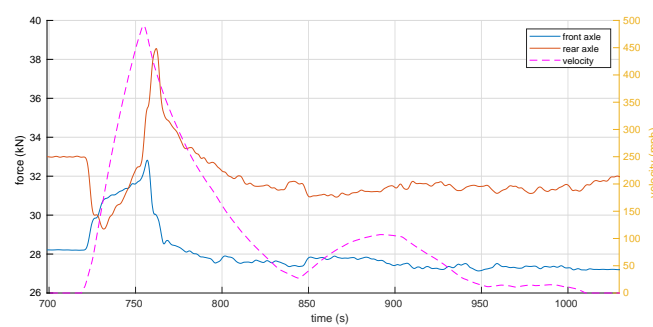

(c) Run 7

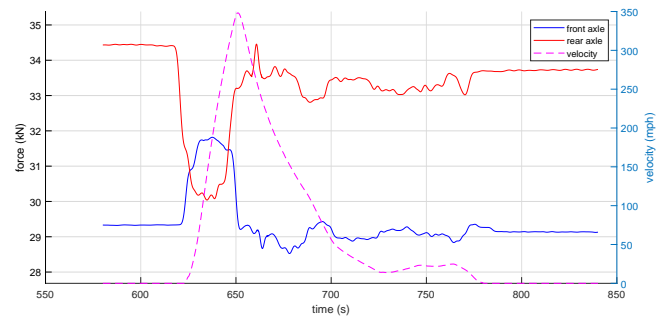

(e) Run 11

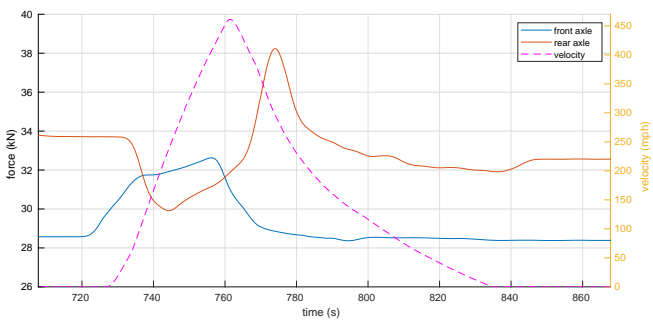

(b) Run 6

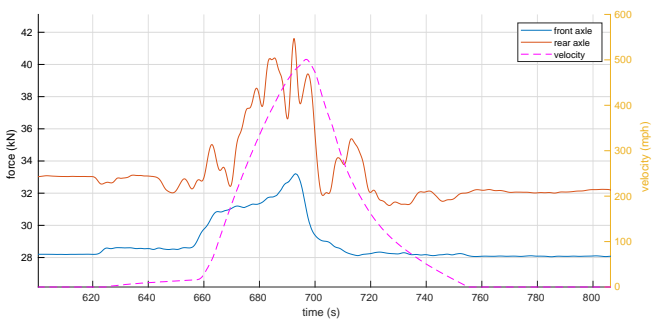

(d) Run 8

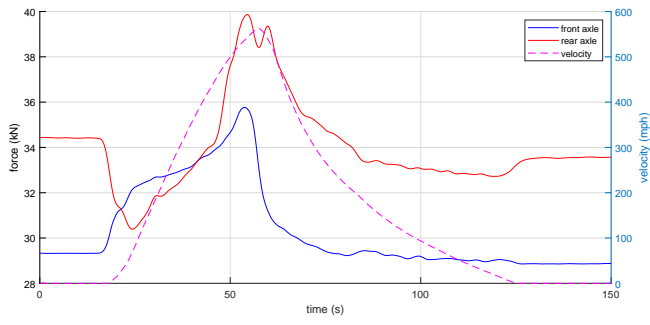

(f) Run 12

Fig. 27 Bloodhound front:rear wheel loading distributions on a range of HST runs

the strong subsonic diffuser downforce under the rear of the car. Flow around the rear wheels and suspension approach and then exceed sonic conditions and the first (weak) shock systems appear leading to a reduction in downforce.

After the jet is throttled back to idle, as peak speed is reached, there is a combination of effects including transfer of load back from the front axle to the rear axle in combination with rear downforce increasing in the early stages of deceleration. Once the point of peak downforce is passed during the deceleration phase the loads on both front and rear axles then reduce back down to the static load distribution values with the car approximately $1.5 \mathrm{kN}$ lighter than at the start of the run due to fuel burn. This wheel load behaviour is in excellent agreement with the net CFD vertical force distribution predictions, after non-aerodynamic factors are taken into account, leading to the conclusion that although there are significant localised discrepancies between CFD predictions and measured pressures, the net, integrated effect of the simulated aerodynamic loading is in good agreement with the overall measured vehicle performance.

The equivalent wheel loading data for runs 5, 6, 7, 8, 11 and 12 are shown in Figure 27 (note that run 9 was aborted and therefore data not analysed). 


\section{B. Laser Rideheight/Pitch Measurements}

Four laser rideheight sensors [45] were positioned at each of the vehicle's wheels to measure the displacement between the underside of the car and the ground plane. Figure 28 shows the raw measurements from these sensors along with a 1 second moving average trace which filters the noise. These sensors only functioned at relatively low speed (below $\approx 350 \mathrm{mph}$ on the front sensors and below $\approx 300 \mathrm{mph}$ on the rear sensors). For this reason only data for the first three HST runs of Bloodhound is shown. Also displayed in Figure 28 is the derived change in body pitch angle throughout these runs. This value was calculated by taking the mean change in front and rear sensor heights and assuming a perfectly flat ground plane.

In each case the rideheight variations are of the order of $5-10 \mathrm{~cm}$ and pitch angle variations of the order 0.2 degrees (note that the vehicle wheel base is $8.9 \mathrm{~m}$ ). The fact that the measured rideheights don't settle back to the same starting positions at the end of a run is likely to be due to variations in wheel penetration which (although not taken into account when deriving pitch) did happen in practice due to variations in surface consistency.

The first installation run of the car used relatively low throttle with a set of gentle accelerations and decelerations. It is not evident, from Figure 28 (a), that there is any meaningful correlation between the car performance and rideheight/pitch changes on this first run. However, in Figures 28 (b) and (c), where full throttle was used during a single acceleration and deceleration the pitching forward of the body on application of thrust and then subsequent pitching up after throttling back to idle is evident. It is interesting to note that, based on the work in [26] which deduced, via CFD, that the pitch sensitivity of normalised lift, $L / q\left(\mathrm{~m}^{2}\right)$, at subsonic speeds was likely to be of the order $1.3 \mathrm{~m}^{2}$ per degree of pitch, that at $300 \mathrm{mph}$ a $0.2^{\circ}$ change in pitch corresponds to a $2.5 \mathrm{kN}$ change in vertical load acting across the entire vehicle $(0.625$ $\mathrm{kN}$ per wheel). When compared to the wheel load variations shown in SectionIV.A the change in loading on the wheels due to pitch at $300 \mathrm{mph}$ is not likely to be have been the dominant influence although perhaps was important at higher speeds (however higher speed vehicle pitch data is not available to test this theory).

\section{Vehicle Dynamics Modelling}

A simple vehicle dynamics performance model was developed in MATLAB Simulink [47] using the default Forward Euler method for time integration. Initial inputs were based on the following assumptions:

- the simple EJ200 jet thrust map to throttle setting was provided by the engine manufacturer and assumed correct (note that absolute thrust values used cannot be provided due to military classification)

- at the start of a run the engine instantaneously increases from idle to max thrust and instantaneously returns to idle at peak speed

- a fixed coefficient of rolling resistance

- a fixed wheel brake decelerating force upon application

- inclusion of a velocity-dependent spray drag term guided by the work in [25] as detailed in Section [III.D 

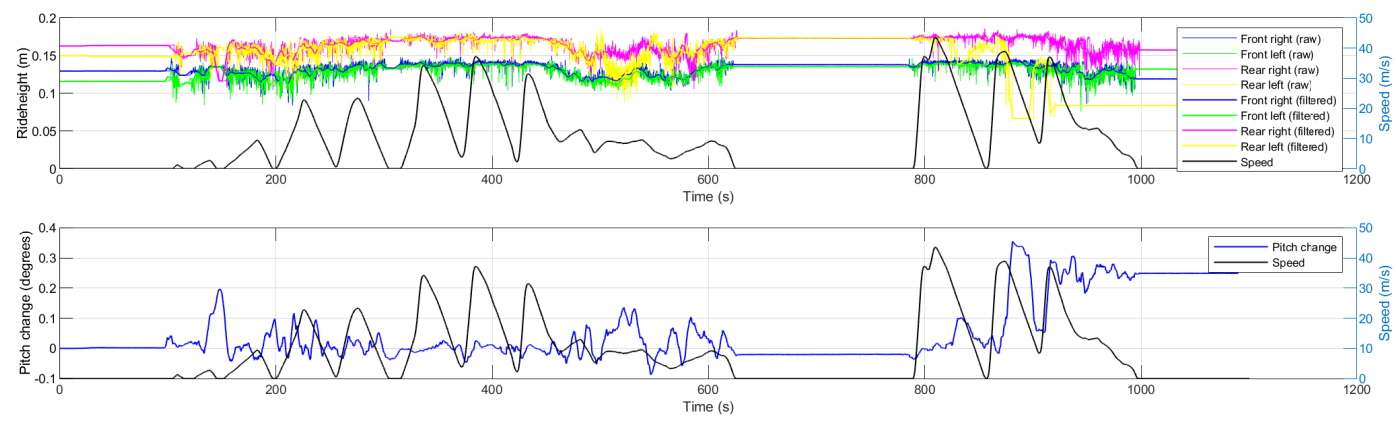

(a) Run 1 (installation run, peak speed $99 \mathrm{mph}$ )
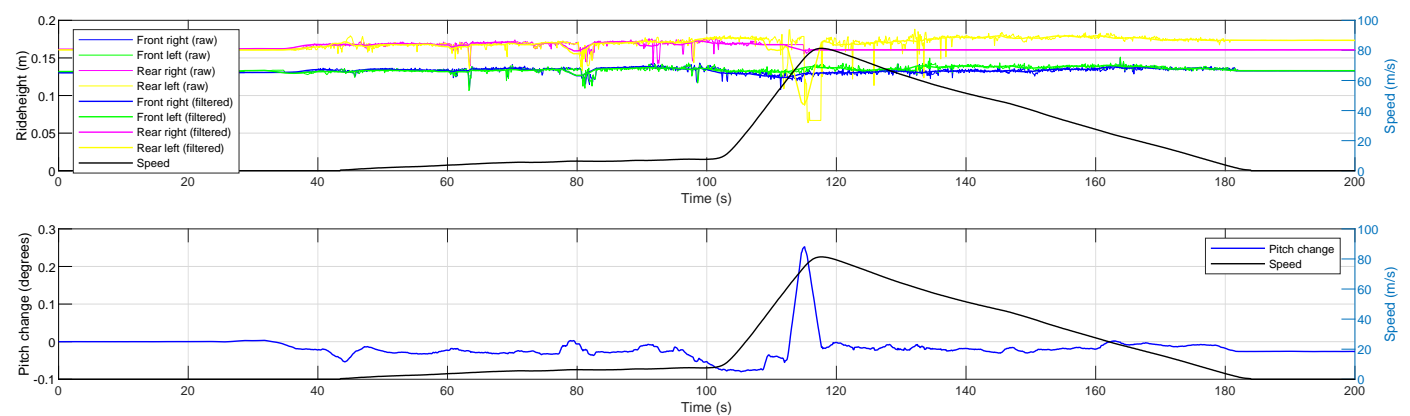

(b) Run 2 (peak speed $190 \mathrm{mph}$ )
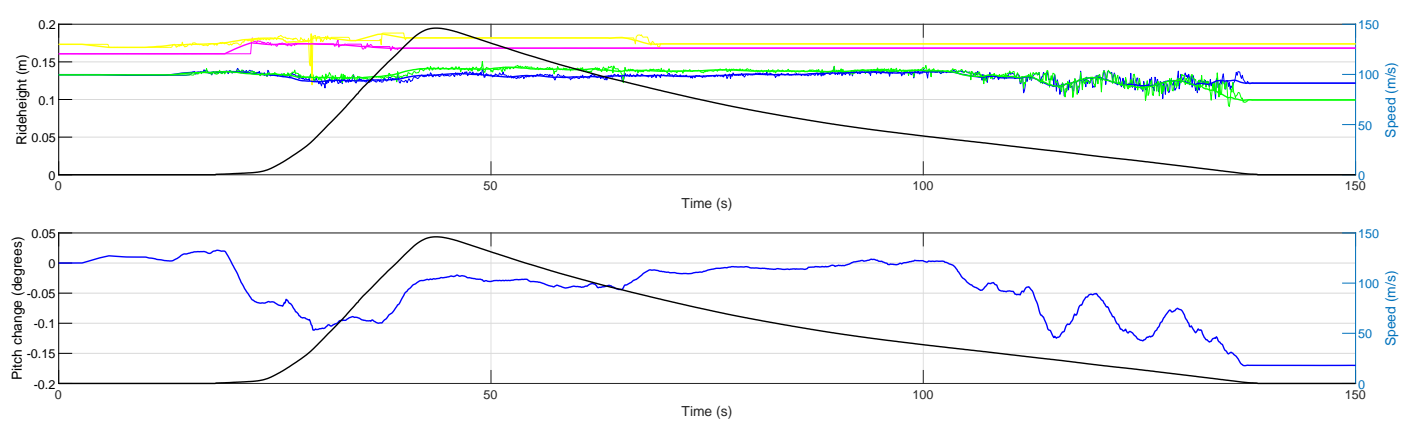

(c) Run 3 (peak speed $334 \mathrm{mph}$ )

Fig. 28 Vehicle laser rideheight measurements on runs 1, 2 and 3. Raw data and 'filtered' data using a 1 second moving average is displayed. Also shown is the derived body pitch angle change throughout the runs. 
- aerodynamic drag figures for both the car body and air brakes came directly from CFD simulation results using HLLC-SST turbulence model with published base drag correction [26]

- deployment of parachute(s) modelled based on a fixed $D_{q}$ term

The model was then tuned after each run of the car to match the actual vehicle performance throughout the lower speed runs to allow an accurate prediction of vehicle performance in the higher speed runs during the HST programme. In order to achieve this the following changes were made to the model based on a trial and error process:

- thrust values were adjusted from the theoretical values provided by the engine manufacture by a linear scale factor to account for any engine under-performance (for example due to ambient air temperature / altitude effects) and intake spill drag

- the coefficient of rolling resistance, spray drag, wheel brake and air brake values were scaled

- the pre-computed CFD predictions for overall vehicle drag were not adjusted to account for the over-prediction of base pressure drop (as discussed in Section III)

\begin{tabular}{llllll}
\hline \multicolumn{2}{c}{ Ambient Conditions } & \multicolumn{2}{c}{ Modelling Parameters } & \multicolumn{2}{c}{ Run Details } \\
\hline Temperature & $25^{\circ} \mathrm{C}$ & Rolling Resistance & 0.11 & Car Mass & $6,500 \mathrm{~kg}$ \\
Pressure & $921 \mathrm{mBar}$ & Braking Decel $(\mathrm{g})$ & 0.08 & Braking Speed & $226 \mathrm{mph}$ \\
Wind & $10 / \mathrm{c}, 3-6 \mathrm{mph}$ & Parachute D/q & 1.7 & Parachute Speed & $590 \mathrm{mph}$ \\
& & Spray Drag (kNs/m) & 0.01 & Peak Speed & $628 \mathrm{mph}$ \\
& & Thrust Data* & $98 \%$ & Track\#/start pos & $3 \mathrm{~S}, \mathrm{~km} 0.2$ \\
\hline
\end{tabular}

Table 4 Bloodhound performance model parameter assumptions. *Theoretical jet thrust scaled by the given percentage.

Figures 30 to 37 show the results from the tuned performance model relative to the actual vehicle performance for runs $2,3,6,7,8,9,11$ and 12 . In these plots the Gross $T_{\text {actual }}$ term is the gross thrust output from the EJ200 jet computer (i.e. the gross thrust that the engine believes it is producing) whilst the Net $T_{\text {model }}$ is the value used based on the engines manufacturer's mapping to throttle setting that attempts to take into account the intake spill drag term and other intake losses. It is important to note that, other than the consistent modification of CFD base drag prediction, the (HLLC-SST) CFD drag predictions resulted in an almost perfect match to the actual vehicle dynamic perforamance across this speed range. This gives good confidence in the effectiveness of RANS-based CFD modelling for applications such as this provided that known issues such as base drag prediction are accounted for.

\section{Airbrake Performance}

HST runs 10 and 11 were undertaken with the vehicle's airbrake doors fully extended throughout the entire run. This was done to establish the braking (drag) performance of the airbrakes as a function of vehicle speed and also to assess the risk of the wake from the airbrakes adversely affecting the structure, in particular the rear wheel and 


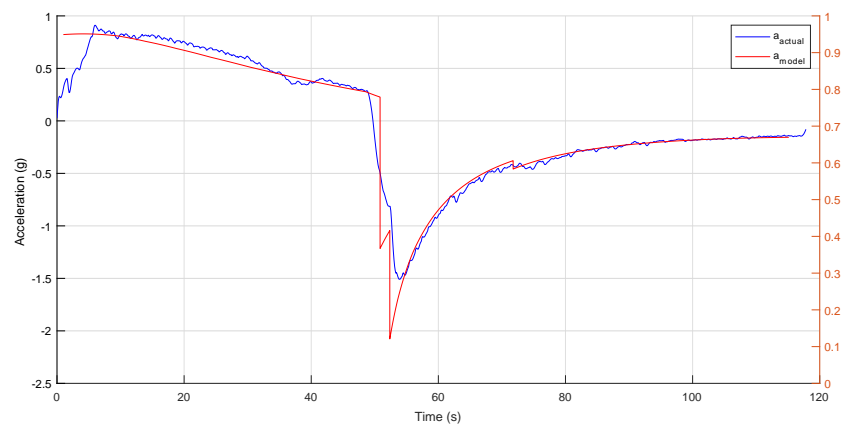

(a) Acceleration against time

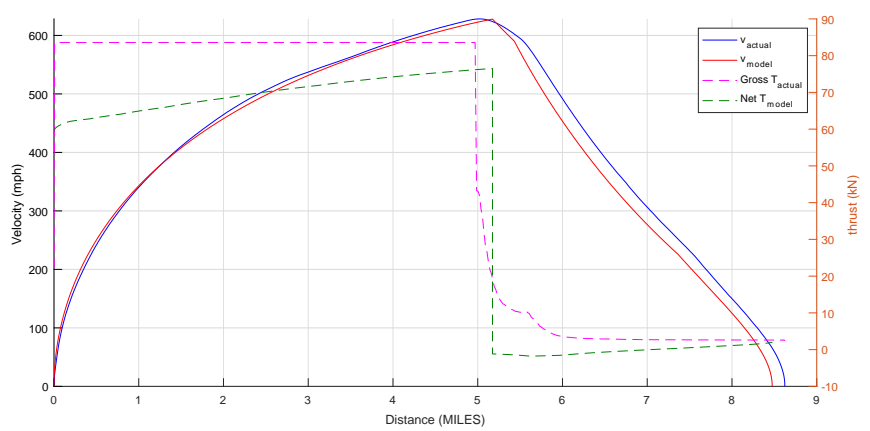

(b) Velocity against distance

Fig. 29 Bloodhound performance model and actual vehicle performance on run 13 


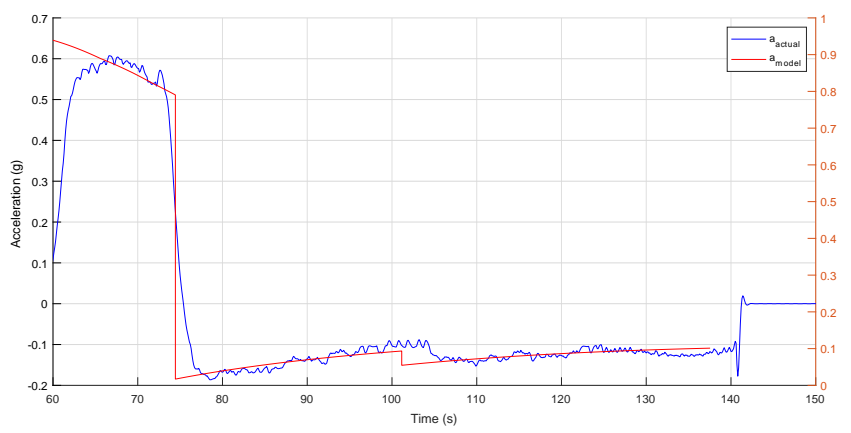

(a) Acceleration against time

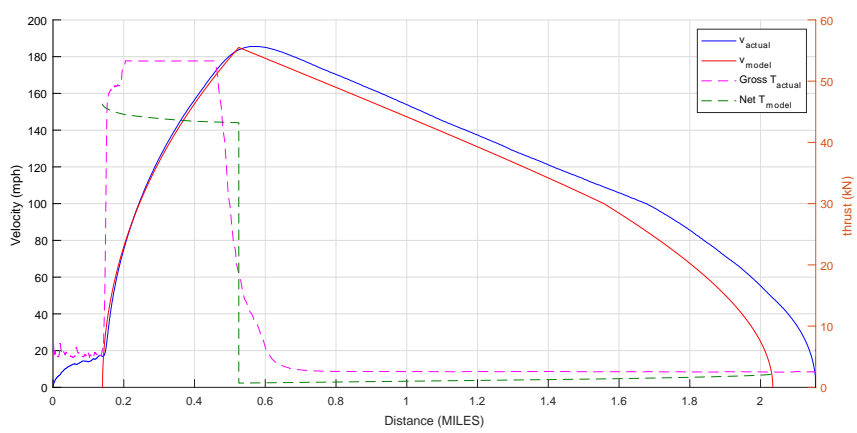

(b) Velocity against distance

Fig. 30 Bloodhound performance model and actual vehicle performance on run 2 


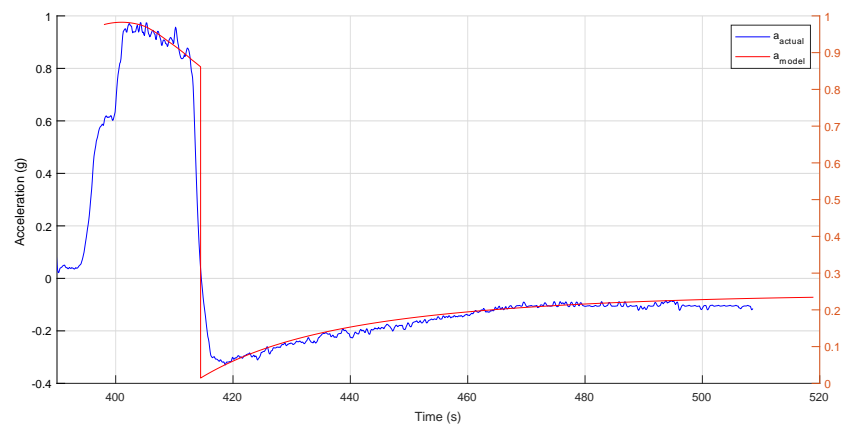

(a) Acceleration against time

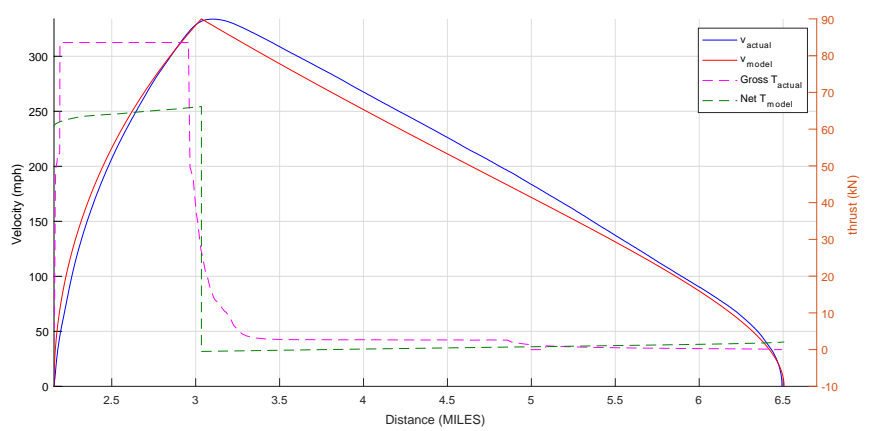

(b) Velocity against distance

Fig. 31 Bloodhound performance model and actual vehicle performance on run 3 


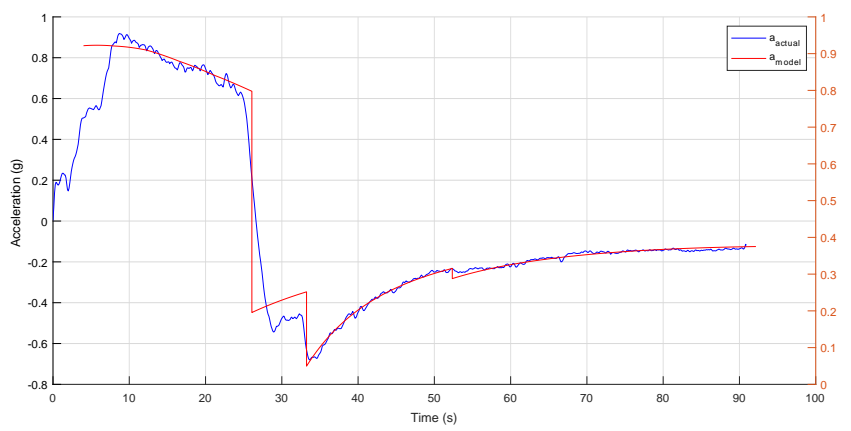

(a) Acceleration against time

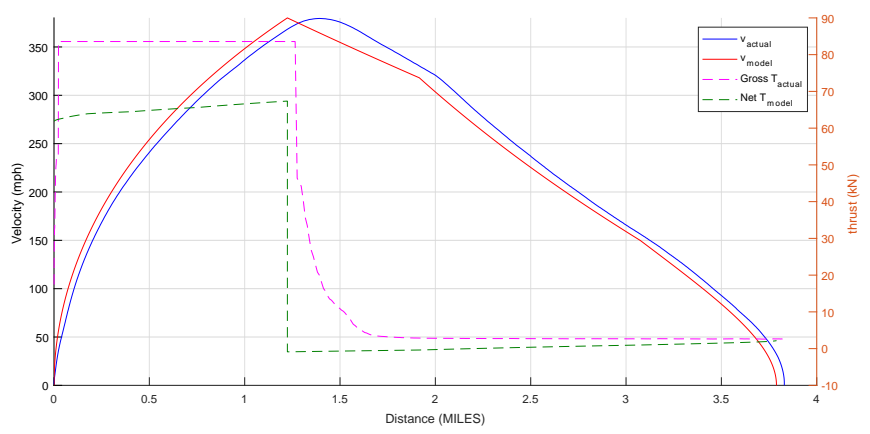

(b) Velocity against distance

Fig. 32 Bloodhound performance model and actual vehicle performance on run 6 


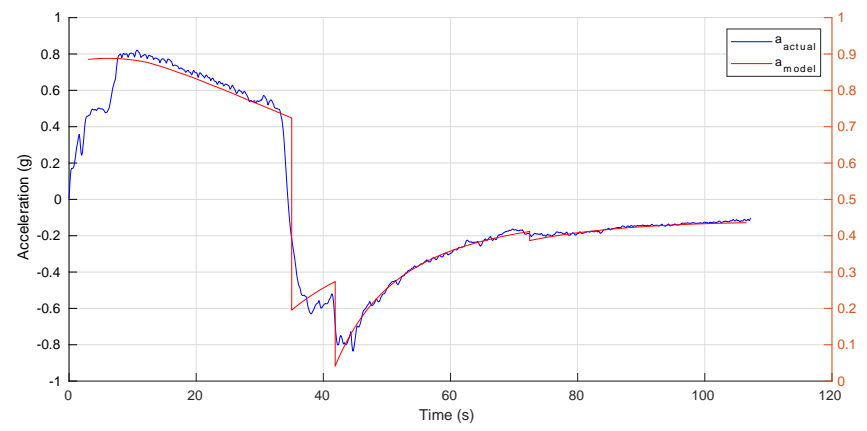

(a) Acceleration against time

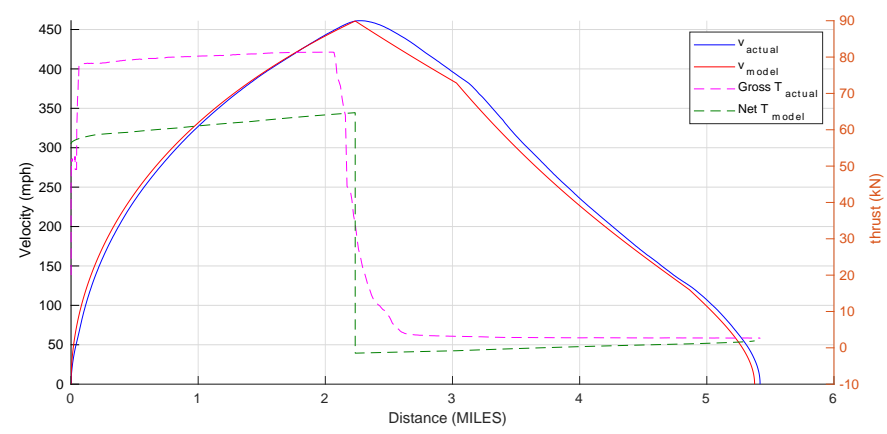

(b) Velocity against distance

\section{Fig. 33 Bloodhound performance model and actual vehicle performance on run 7}

suspension geometry, downstream. Previous CFD analysis of the airbrake had been conducted [4] to determine the likely aerodynamic drag of the airbrakes. This work led to the introduction of the perforations in the design, seen in Figure 38, which have the effect of reducing the size and increasing the frequency of the shedding vortex structures in the wake to above the natural frequency of the rear vehicle structure.

In order to match the actual vehicle performance in the airbrake extended runs 10 and 11 a normalised drag $(D / q)$ value of $1.3 \mathrm{~m}^{2}$ was required compared with the value of $1.4 \mathrm{~m}^{2}$ predicted by the airbrake CFD model [4], again implying that the CFD was slightly over-predicting the drag. Strain gauges positioned on the airbrake actuation struts measured a peak streamwise (drag) load of 14kN during run 11 (at $350 \mathrm{mph}$ ). This, in absolute load terms, is slightly lower than the $17.5 \mathrm{kN}$ predicted by the CFD model at this speed. This could be due to the CFD model over-predicting base drag on the airbrake as was the case on the car body base (although no pressure sensors were located on the airbrake doors to verify this) or because of the fact that airbrake CFD modelling was conducted on a reduced geometry with airbrakes positioned against a flat plate rather than the actual vehicle geometry. However, overall, the RANS CFD predictions of drag on the airbrakes provided an excellent estimate of the actual aerodynamic performance. Accurate corrections can now be made to the CFD predictions for future, higher speed, airbrake deployments.

There was no obvious damage or detrimental effects on the vehicle structure downstream of the airbrakes on either 


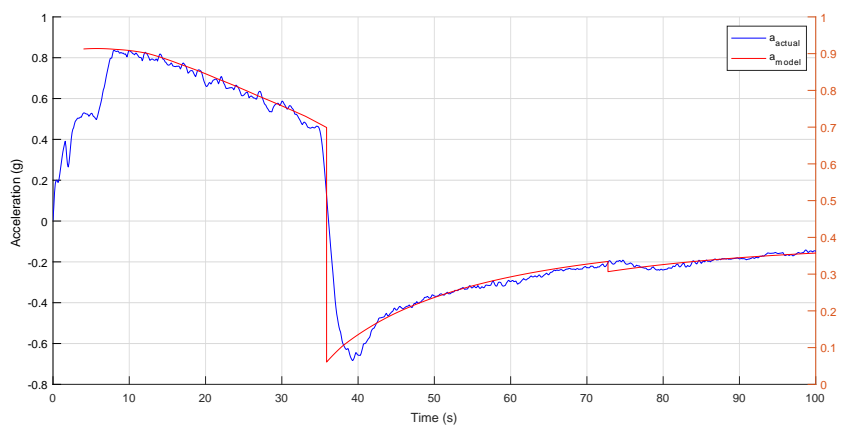

(a) Acceleration against time

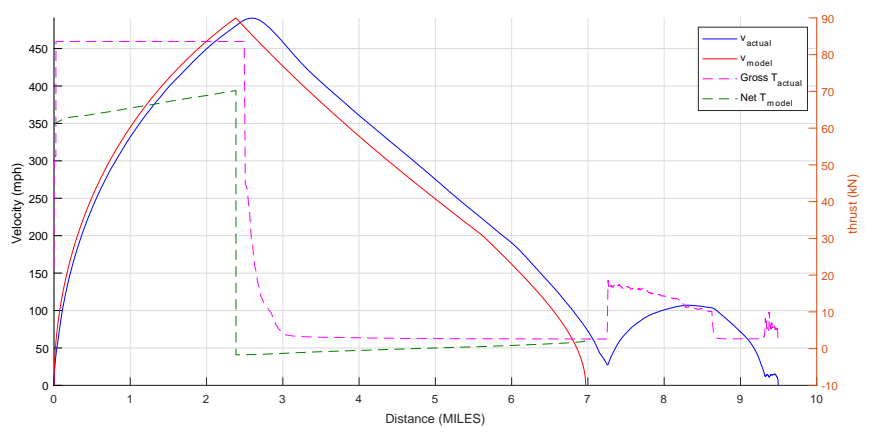

(b) Velocity against distance

Fig. 34 Bloodhound performance model and actual vehicle performance on run 8 


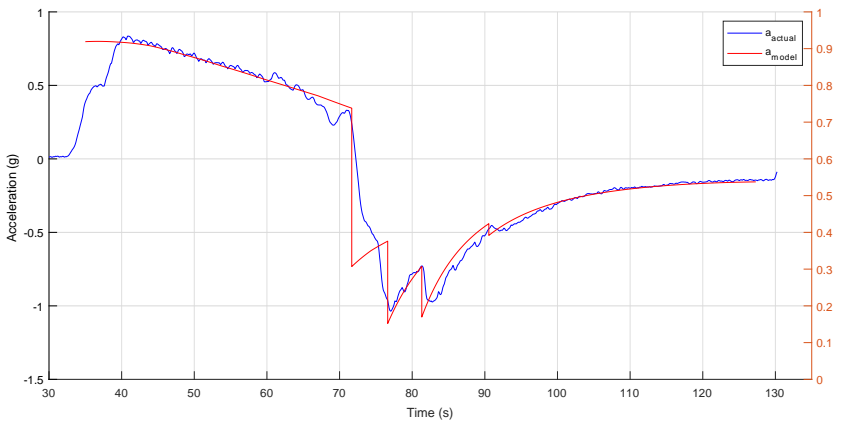

(a) Acceleration against time

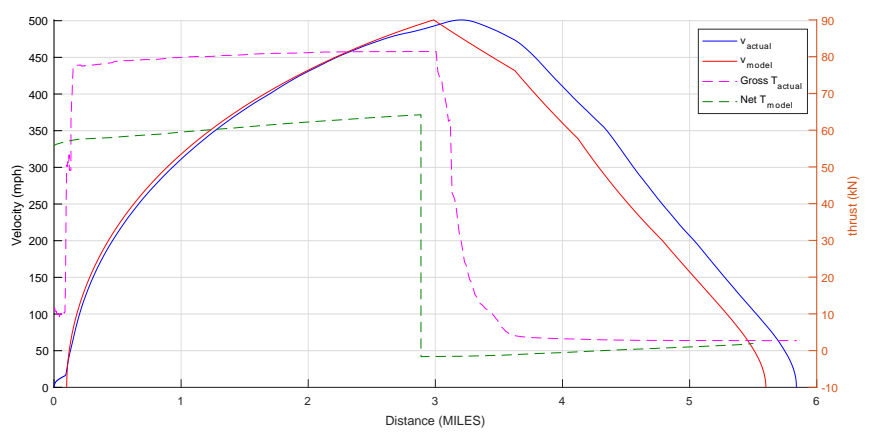

(b) Velocity against distance

Fig. 35 Bloodhound performance model and actual vehicle performance on run 9 


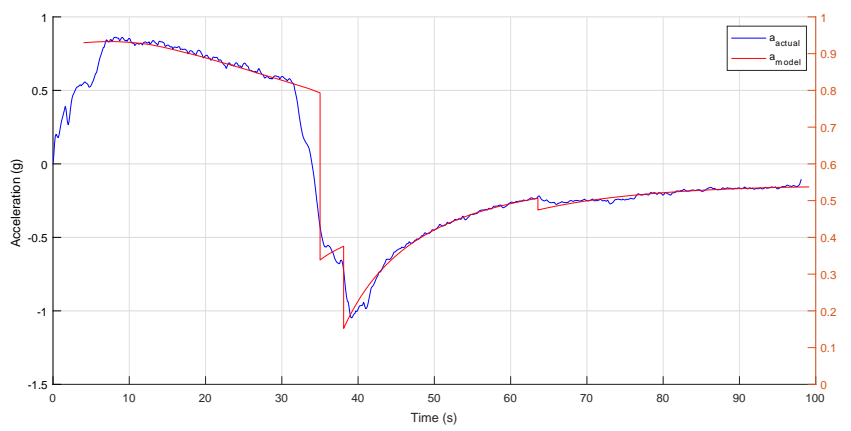

(a) Acceleration against time

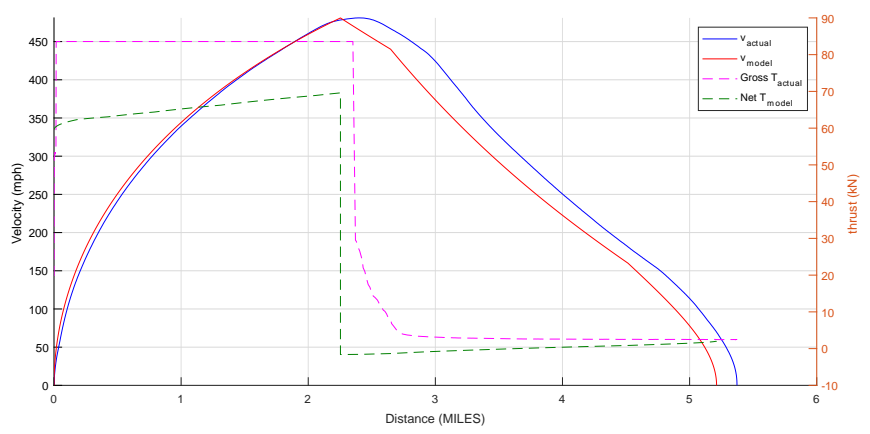

(b) Velocity against distance

Fig. 36 Bloodhound performance model and actual vehicle performance on run 11 


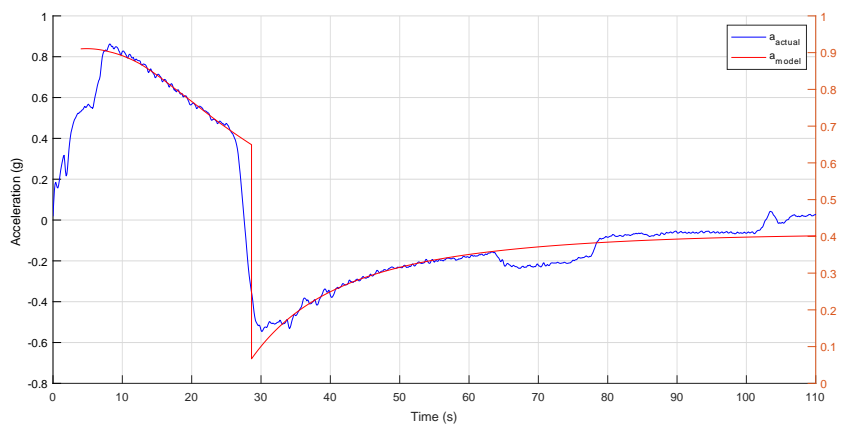

(a) Acceleration against time

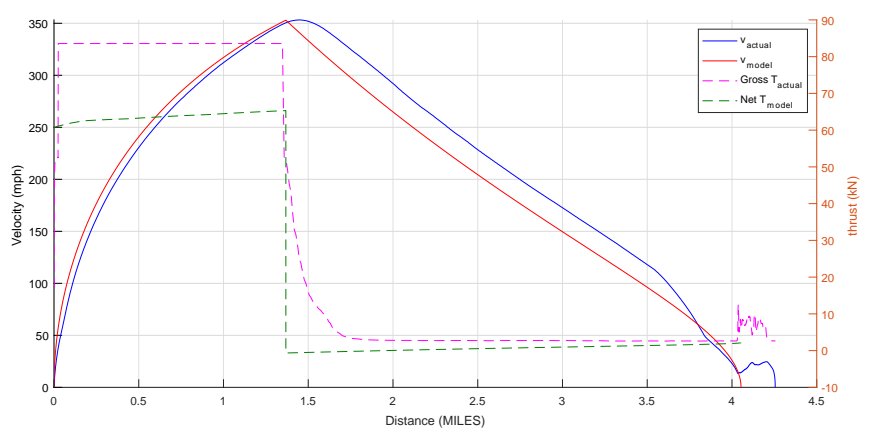

(b) Velocity against distance

Fig. 37 Bloodhound performance model and actual vehicle performance on run 12 

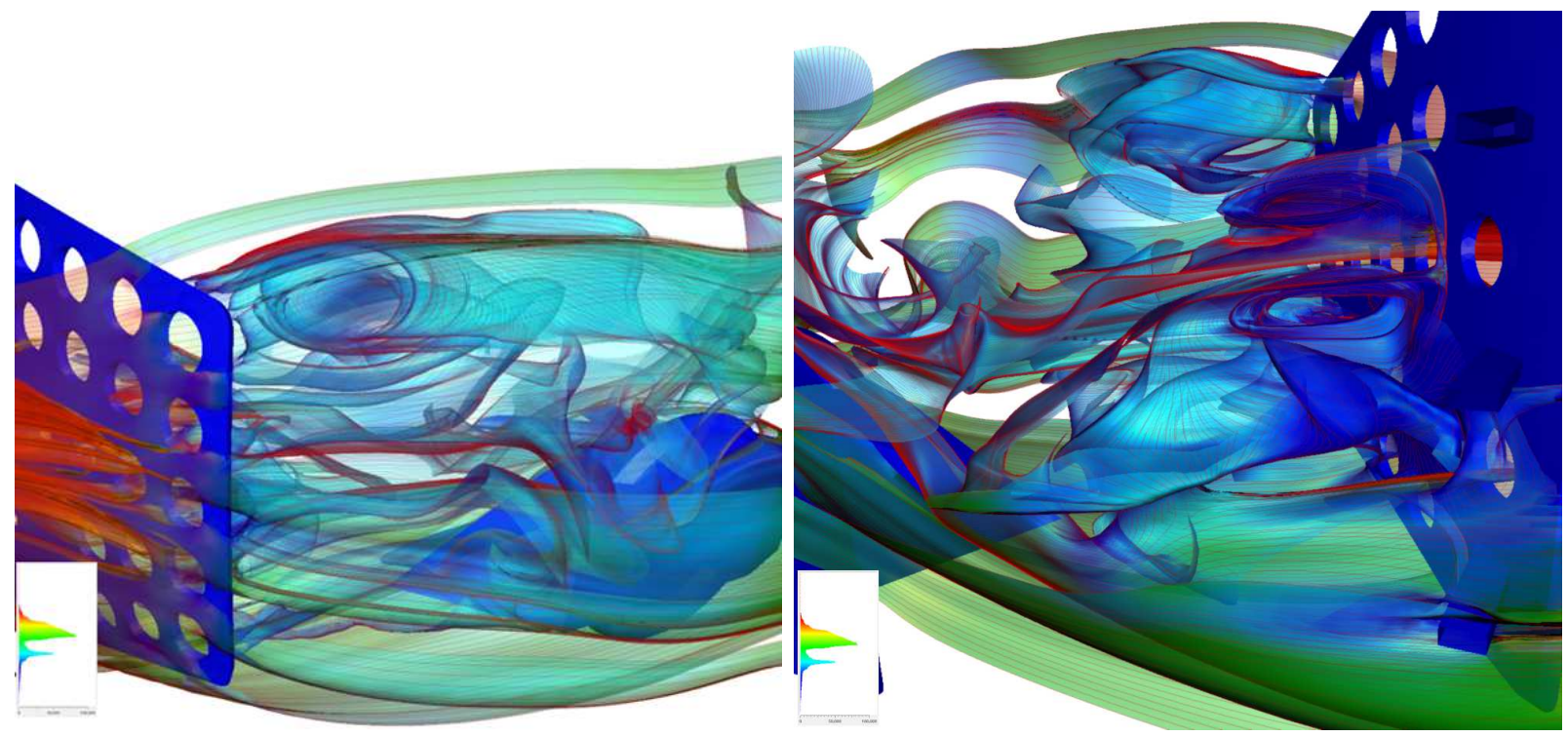

(a) CFD visualisation of flow downstream of airbrake deployed at 60 (b) CFD visualisation of flow downstream of airbrake deployed at 60 degrees at Mach 0.5 [46 degrees at Mach 0.5 [46
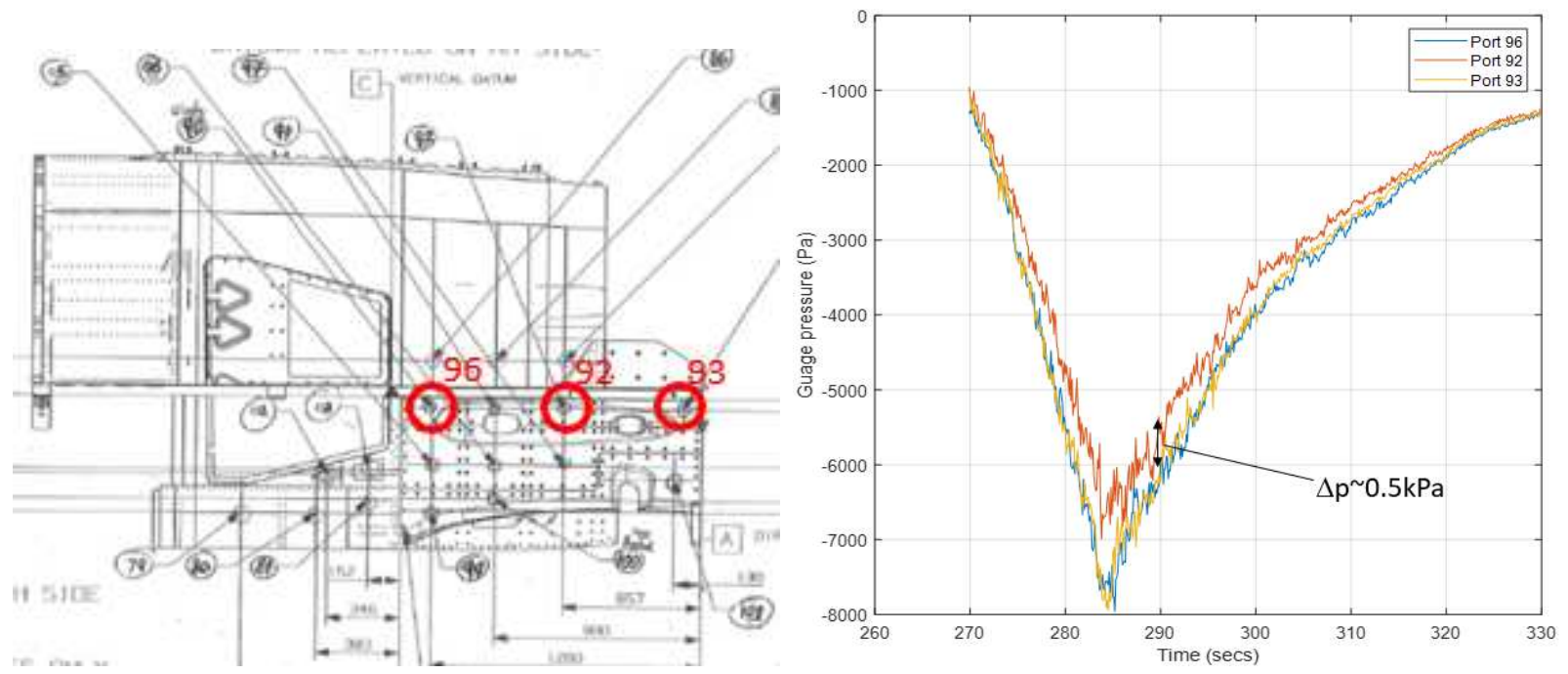

(c) Sensor locations...

(d) N.B. Data captured at $10 \mathrm{~Hz}$, typical aerodynamic oscillation frequencies being captured here $1-2 \mathrm{~Hz}$

Fig. 38 Airbrake performance - simulated and measured 
run 10 or 11 indicating that the airbrake wake was not exciting the structure. Data from pressure sensors located in the wake region immediately downstream of the airbrakes is shown in Figure 38(d) although it must be stressed that this data was captured at $10 \mathrm{~Hz}$ so higher frequency wake features are not evident.

\section{Conclusions and Recommendations}

This paper has outlined the main aerodynamic and vehicle performance findings from the high speed testing phase of the development of the Bloodhound Land Speed Record vehicle. From extensive analysis of the data obtained over 13 runs of the vehicle at speeds of up to $628 \mathrm{mph}$ (Mach 0.81) the following set of conclusions and recommendations have been deduced.

Firstly, related to the CFD modelling used in previous works [] to predict the aerodynamic characteristics of the vehicle:

- Steady RANS CFD approaches can provide aerodynamic performance predictions on accelerating, complex geometry bodies in ground-effect to a sufficient degree of accuracy to allow reasonable prediction of overall aerodynamic loads and moments acting on the vehicle even beyond the critical Mach number.

- Localised aerodynamic and wheel-ground interaction aerodynamic phenomena such as particle entrainment and a non-isotropic surface consistency are still a significant challenge for conventional CFD methods.

- At transonic speeds the RANS approach used in this work tended to over-predict base drag due to an exaggerated prediction of base pressure drops.

- The RANS approach used in this work tended to over-predict the drag on geometries with large levels of flow separation (i.e. the airbrake doors)

- The impact of the choice of turbulence model has a relatively small impact on the accuracy of the CFD predictions but, on average, the SST turbulence model tended to better predict the measured pressure distributions than the SA model across the speed range considered.

Secondly, with regards the overall performance of Bloodhound:

- The understanding that the data presented in the paper provides, particularly with regards to the maps of thrust and drag as a function of vehicle speed, has allowed the engineering team to determine the rocket system specification (i.e. required specific impulse) to allow a $800 \mathrm{mph}$ record attempt to be conducted and a, hypothetical, 1,000 mph attempt.

- The wheel loading measurements map well predictions made using CFD data over the speed range considered during high speed testing. This gives the engineering team confidence that, with the addition of small fin-mounted winglets to trim vertical loads at the rear of the car, the basic design of Bloodhound is safe to continue with the project and attempt a $800 \mathrm{mph}$ Land Speed Record.

- A 1,000 mph Land Speed Record is still hypothetically possible but would require more data up to low supersonic 
speeds to provide sufficient confidence that it could be attempted safely within the track limit available at Hakskeen Pan.

And finally, with regards to potential design improvements to the car to improve performance for a future record attempt:

- Minimisation of spray drag and mitigation against particle impact damage might be possible by the introduction of longitudinal strakes on the underside edge of the car body to minimise the spill of particles from underneath the main vehicle body.

- The base drag of the vehicle could be reduced further by tapering the rear delta fairings and lower main body subject to this being possible without introducing unwanted vertical aerodynamic loading.

Overall, the high speed test programme for the Bloodhound Land Speed Record car in 2019 was deemed a success. It has provided the engineering team with sufficient confidence that an $800 \mathrm{mph}$ Land Speed Record is possible and, at the time of writing, the team is preparing to return to South Africa for a record attempt.

\section{Acknowledgments}

The authors would like to thank all of the students and supporters who have contributed to the aerodynamic modelling and design work of Bloodhound over the course of the project. Also the authors would like to acknowledge the team of engineers, investors and sponsors who have made the Bloodhound project a reality.

The initial CFD development work necessary for this project was conducted under EPSRC grant EP/F032617.

\section{References}

[1] WWW.bloodhoundlsr.com accessed on 3rd December 2020

[2] Morgan K, Hassan O, Weatherill N. MATHEMATICS-Why Didn’t The Supersonic Car Fly?. Mathematics Today-Bulletin of the Institute of Mathematics and its Applications. 1999;35(4):110-4.

[3] Lock A. Computational fluid dynamics development of the JCB DIESELMAX land speed record vehicle. SAE Technical Paper; 2007 Sep 17.

[4] Evans BJ, Hassan O, Jones JW, Morgan K, Remaki L. Computational fluid dynamics applied to the aerodynamic design of a land-based supersonic vehicle. Numerical Methods for Partial Differential Equations. 2011 Jan;27(1):141-59.

[5] Doig G. Transonic and supersonic ground effect aerodynamics. Progress in Aerospace Sciences. 2014 Aug 1;69:1-28.

[6] Mach, E. and Wosyka, J., 1875. Über einige mechanische Wirkungen des elektrischen Funkens. Annalen der Physik, 232(11), pp.407-416.

[7] Wieselsberger, C., 1922. Wing resistance near the ground. NACA TM-77. 
[8] Barber TJ, Leonardi E, Archer RD. Causes for discrepancies in ground effect analyses. The Aeronautical Journal. 2002 Dec;106(1066):653-68.

[9] Willemsen E. High Reynolds number wind tunnel experiments on trains. Journal of wind engineering and industrial aerodynamics. 1997 Jul 1;69:437-47.

[10] Baker CJ, Brockie NJ. Wind tunnel tests to obtain train aerodynamic drag coefficients: Reynolds number and ground simulation effects. Journal of Wind Engineering and Industrial Aerodynamics. 1991 Jun 1;38(1):23-8.

[11] Kleine H,Hiraki K,Oakes B,Young J,Kusano H,Iritani Y., 2011. Projectiles in transonic ground effect. Proceedings of the 29th International congress on high-speed imaging and photonics.

[12] Major I, R\&D ranges and test facilities:summary of capabilities. United States Army Test and Evaluation Command ADA265938;1990

[13] Nakata D, Yajima J, Nishine K, Higashino K, Tanatsugu N, Kozu A. Research and development of high speed test track facility in Japan. In50th AIAA aerospace sciences meeting including the New Horizons forum and aerospace exposition 2012 (p. 928 ).

[14] Nakata D, Kozu A, Yajima J, Nishine K, Higashino K, Tanatsugu N. Predicted and experimented acceleration profile of the rocket sled. TRANSACTIONS OF THE JAPAN SOCIETY FOR AERONAUTICAL AND SPACE SCIENCES, AEROSPACE TECHNOLOGY JAPAN. 2012;10(ists28):1-5.

[15] Minto D. CTEIP funded advances in hypersonic testing at the Hollman high speed test track. In24th AIAA Aerodynamic Measurement Technology and Ground Testing Conference 2004 Jun 28 (p. 2740).

[16] Noble R, Tremayne D. Thrust(text). Partridge Press; 1-8522-5268-5.

[17] Ayres R, Thrust SSC. Environmental testing. Environ Eng 1996; 9(2):28-30

[18] Haddleton GP. Use of high-speed photography and associated techniques as a measurement tool in the research, development, test, and evaluation of weapons systems and armaments. In21st International Congress on: High-Speed Photography and Photonics 1995 May 30 (Vol. 2513, pp. 782-791). International Society for Optics and Photonics.

[19] Katz J. Aerodynamics of race cars. Annu. Rev. Fluid Mech.. 2006 Jan 21;38:27-63.

[20] Torda TP, Uzgiris SC. Blue flame-A land speed record vehicle. Mechanical Engineering. 1970 Jan 1;92(7):9.

[21] Keogh J, Doig G, Diasinos S. Flow compressibility effects around an open-wheel racing car. The Aeronautical Journal. 2014;118(1210):1409.

[22] Keogh J, Doig G, Diasinos S. The influence of compressibility effects in correlation issues for aerodynamic development of racing cars. AFMS. 2012 Dec 3.

[23] Oberoi R, Chakravarthy S, Fredrick W, Glessner PT. Using CFD to design the American challenger rocket car. SAE Technical Paper; 2006 Dec 5. 
[24] Hwang JP,Doig G, Barber TJ, Neely AJ, Leonardi E. Numerical investigation of the aerodynamics of land speed record vehicles. Proceedings of the 17th international shock interaction symposium, Rome;3-7 September 2006

[25] Remaki L, Hassan O, Evans BJ, Morgan K. Spray drag effect of fluidized sand for a supersonic vehicle. Journal of Coupled Systems and Multiscale Dynamics. 2014 Oct 1;2(3):169-77.

[26] Evans B, Rose C. Simulating the aerodynamic characteristics of the Land Speed Record vehicle BLOODHOUND SSC. Proceedings of the Institution of Mechanical Engineers, Part D: Journal of Automobile Engineering. 2014 Sep;228(10):1127-41.

[27] Evans B, Morton T, Sheridan L, Hassan O, Morgan K, Jones JW, Chapman M, Ayers R, Niven I. Design optimisation using computational fluid dynamics applied to a land-based supersonic vehicle, the BLOODHOUND SSC. Structural and Multidisciplinary Optimization. 2013 Feb 1;47(2):301-16.

[28] Townsend J, Evans B, Tudor T. Aerodynamic optimisation of the rear wheel fairing of the land speed record vehicle BLOODHOUND SSC. The Aeronautical Journal. 2016 Jun;120(1228):930-55.

[29] https://www.dendrite.me/media/output/mediaid/54e214dc861dc6cf73e517a2 accessed 3rd December 2020

[30] Kleine, Flow Visualization, Handbook of Shock Waves (editors: G. Ben Dor, O. Igra, T. Elperin), vol. 1, ch. 5.1, pp. 683-740, Academic Press, San Diego, 2001

[31] https:/www. aussieinvader.com accessed on 3rd December 2020

[32] Raghunathan RS, Kim HD, Setoguchi T. Aerodynamics of high-speed railway train. Progress in Aerospace sciences. 2002 Aug 1;38(6-7):469-514.

[33] Holloran M, O’Meara S. Wing in ground effect craft review. Defence Science and Technology Organisation Canberra (Australia); 1999 Feb 1.

[34] Kleine H, Oakes B, Pratt H, Neely AJ, Barber TJ. Shock wave interaction with gaseous plumes and flames. In29th International congress on high-speed imaging and photonics proceedings, ICHSIP-29 2010 (pp. 1-6).

[35] Altmann J. Acoustic weapons-a prospective assessment.Science and Global Security. 2001 Jan 1;9(3):165-234.

[36] Roohani H, Skews BW. The influence of acceleration and deceleration on shock wave movement on and around aerofoils in transonic flight. Shock Waves. 2009 Aug 1;19(4):297-305.

[37] Paton RT, Skews BW. Shock and Blast Wave Interaction with Hard Sand Pan. InInternational Symposium on Shock Waves 2017 Jul 9 (pp. 673-679). Springer, Cham.

[38] Wilcox DC. Formulation of the kw turbulence model revisited. AIAA journal. 2008 Nov;46(11):2823-38.

[39] Menter FR. Two-equation eddy-viscosity turbulence models for engineering applications. AIAA journal. 1994 Aug;32(8):1598605. 
[40] Spalart P, Allmaras S. A one-equation turbulence model for aerodynamic flows. In30th aerospace sciences meeting and exhibit 1992 Jan 6 (p. 439).

[41] Jameson A, Schmidt W, Turkel E. Numerical solution of the Euler equations by finite volume methods using Runge Kutta time stepping schemes. In14th fluid and plasma dynamics conference 1981 Jun 23 (p. 1259).

[42] Anderson JD. Introduction to Aerodynamics. High speed aerodynamics. 2007.

[43] Tinoco, E.N. et al, Summary Data from the Sixth AIAA CFD Drag Prediction Workshop: CRM Cases, Journal of Aircraft, 55 (4), pp 1352-1379, 2018

[44] Corke TC. Design of aircraft. Englewood Cliffs, NJ: Prentice Hall; 2003.

[45] https://wWw.gillsc.com/accessed on 3rd December 2020

[46] Edmunds M, Evans B, Masters I, Laramee RS. Enhanced flow visualisation of complex aerodynamic phenomena using automatic stream surface seeding with application to the BLOODHOUND SSC Land Speed Record vehicle. The Aeronautical Journal. 2016 Apr;120(1226):547-71.

[47] https://uk.mathworks.com/products/simulink.html accessed on 3rd December 2020 